\title{
Unscharfe Grenzen von Policy-Feldern im parlamentarischen Diskurs. Messungen und Erkundungen durch korpusunterstützte Politikforschung
}

\section{Kurzfassung}

Mit dem Konzept des „Politikfelds“ und verwandten Grundbegriffen ist die Vorstellung eines politischen Handlungsbereichs mit geklärten Grenzen verbunden. Doch man kann oftmals strittige, unscharfe und überlappende Grenzen beobachten. Handelt es sich dabei um Ausnahmeerscheinungen oder um ein systematisch auftretendes Phänomen? Um Letzteres nachzuweisen, wird ein korpusunterstützter Forschungsansatz verfolgt. Grundlage der Untersuchung ist ein Plenardebattenkorpus, das alle Debatten im nordrhein-westfälischen Landtag der 14. und 15. Wahlperiode umfasst (2005-2012). Auf der Grundlage der Frequenzen von Feldmarkern in entsprechend einer inhaltlichen Klassifikation gebildeten Subkorpora wird für 24 Policy-Felder ein Konzentrationsmaß berechnet, das einen Vergleich ermöglicht, welche Felder stärker konzentriert und welche stärker dezentiert sind. Die Korpusanalyse ermöglicht zugleich die Gewinnung von Zeitreihendaten und gibt Hinweise auf Konzentrations- bzw. Ausdifferenzierungsprozesse. Das methodologische Plädoyer lautet, korpusunterstützte Forschung für die Politikwissenschaft weiter zu erschließen, wenn große Massen digitalen Texts zu bewältigen sind. Für die Theoriebildung der Policy-Analyse ergibt sich die Forderung, Unschärfen der Grenzen von Policy-Feldern, das heißt dem Auftreten von überlappenden oder dezentrierten Feldern systematisch nachzugehen. 


\section{Inhalt}

1. Unscharfe Grenzen als Anomalie? 36

2. Policy-Felder und Bedeutungszuweisung durch Feldmarker 39

a) Die Herstellung von Feldbezügen durch Feldmarker 40

b) Feldmarker in nordrhein-westfälischen Plenardebatten 42

3. Konzentration, Überlappung und Dezentrierung von Policy-Feldern 45

a) Korpus und Datenbank als Ressourcen 45

b) Die Messung von Ausdifferenzierung, Überlappung und Dezentrierung

c) Zur Klassifikation der Plenardebatten 50

4. Konzentration und Dezentrierung bei 24 Policy-Feldern 52

a) Konzentration und Streuung: Synchrone Untersuchung 53

b) Konzentrationsprozesse und Zeitreihen $\quad 55$

c) Textorientierte Detailanalyse: Sozialpolitische Grenzprozesse des Jahres 2008

5. Die korpusunterstützte Bewältigung der Flut digitaler Texte 62

\section{Unscharfe Grenzen als Anomalie?}

In der Politikwissenschaft geht man regelmäßig implizit oder explizit davon aus, dass die Auseinandersetzung mit politischen Sachfragen in ausdifferenzierten Arenen des politischen Handelns erfolgt. Diese Arenen werden unterschiedlich bezeichnet. Die Rede ist unter anderem von „Sektoren“, „Policy-Subsystemen“, „Policy-Netzwerken“ und „Policy-Domains“ (vgl. Burstein 1991; McCool 1998). Der Begriff des „Politikfelds“ ist dabei in der deutschen Policy-Forschung der am weitesten verbreitete Grundbegriff zur Bezeichnung jener politischen Handlungsfelder, welche die Orte der politischen Auseinandersetzung um politische Sachfragen sind. Einschlägige Einführungen und Lehrbücher dieser Teildisziplin tragen den Begriff der „Politikfeldanalyse“ in ihren Titeln (Schneider/Janning 2006; Blum/Schubert 2008; Schubert/Bandelow 2009).

Der grundsätzliche Gehalt der Annahme, dass die auf Policies bezogene Interaktion von Akteuren in Feldern erfolgen, die nicht unbegrenzt sind, sei unbestritten. Die Empirie wirft allerdings Fragen auf, ob und inwieweit Grenzen tatsächlich eindeutig und geklärt sind. Politische Akteure stufen zumindest einige Politikbereiche regelmäßig als Felder mit „Querschnittscharakter“ ein. Derartige Aussagen finden sich etwa in der Integrations-, Verbraucherschutz-, Umwelt-, Klima-, Energie- und 
Mittelstandspolitik - also keineswegs nur ausnahmsweise (vgl. Abschnitt 4.a). Gerade im englischsprachigen Raum wurde die Forderung der gezielten Gestaltung von Politikbereichen mit Querschnittscharakter nachdrücklich erhoben: Das zunächst von der Labour-Regierung Tony Blairs in Großbritannien propagierte Konzept des ,Joined-Up Government“ (Ling 2002; Pollitt 2003; Bogdanor 2005) findet unter der Bezeichnung des „Whole-Of-Government"-Ansatzes verwaltungspraktisch Verbreitung und wird als solcher diskutiert (Christensen/Lægreid 2007). Im Vordergrund steht bei der verwaltungswissenschaftlichen Sichtweise, wie administrative Koordination in Politikbereichen mit Querschnittscharakter erreicht werden kann (vgl. Jantz/Veit 2011). Aus Sicht der Policy-Analyse schließt daran aber auch die grundlegende Frage an, ob nicht eine der Vorstellung des ausdifferenzierten Politikfelds verhaftete Begrifflichkeit ${ }^{1}$ den analytischen Blickwinkel der PolicyForschung über möglichweise entscheidende Grenzprozesse hinweglenkt. Die hier vorgestellten Auswertungen sollen einen Beitrag leisten zu der von Frank Janning geforderten Auseinandersetzung mit den „Grundfragen, was Politikfelder eigentlich sind, wie man sie abgrenzen und erfassen kann und welche internen wie externen Faktoren und Bedingungen auf sie einwirken und in ihnen Veränderungen bewirken“ (Janning 2011: 17).

Richtet sich das Interesse neben dem ausdifferenzierten Politikfeld mit vorläufig geklärten Grenzen auch auf grenzüberschreitende Policy-Felder, ${ }^{2}$ so wird man davon ausgehen müssen, dass solche Felder soziale Konstrukte sind und es keine Möglichkeit des politikwissenschaftlichen Beobachters gibt, das Wesen und den definitorischen Kern der Policy-Felder entkoppelt von den Bedeutungszuweisungen der Akteure zu bestimmen. Unschärfen der Grenzen von Policy-Feldern entstehen, wenn Akteure bei der Auseinandersetzung um politische Entscheidungsmaterien Sinnbezüge zu mehr als einem Policy-Feld herstellen. Die Beschäftigung mit der Formation von Policy-Feldern führt zur Annahme der diskursiven Konstruktion dieser Handlungsbereiche und siedelt sich damit (meta-)theoretisch bei jenen kon-

1 Annahmen der Differenzierungstheorie haben wesentlich zur Fokussierung auf „Politikfelder“ bzw. „Policy-Subsysteme“ beigetragen. Im Widerstreit zwischen systemtheoretischer und akteurszentrierter Differenzierungstheorie wird zwar (Aus-)Differenzierung unterschiedlich erklärt (vgl. Schimank 1985, 2007), diese bleibt aber letztlich als zu erklärendes Phänomen unstrittig. Ungeklärte Grenzen oder gar Querschnittspolitik sind bei diesem theoretischen Blickwinkel von nachrangigem Interesse oder nur Übergangsphänomene.

2 Mit dem Begriff des „Policy-Felds“ wird bewusst das „Feld“ akzentuiert. Diese Akzentuierung stellt eine Querverbindung zur Feldtheorie Bourdieus (Bourdieu 1998), der Theorie der Felder des soziologischen Neoinstitutionalismus (DiMaggio/Powell 1983) und vor allem der jüngst von Fligstein und McAdam vorgelegten Feldtheorie her (2012). Eine feldtheoretisch fundierte Analyse von PolicyFeldern betont Prozesse der Konstruktion ihrer Grenzen, ist aber noch zu entwickeln. 
struktivistischen Ansätzen an, welche die konstitutive Bedeutung von Wissen bzw. Diskursen auch für Fragen der Policy-Analyse betonen (Nullmeier/Rüb 1993; Fischer/Forrester 1993; Fischer 2003). Zu einer Ursache unscharfer Grenzen wird bei dieser Sichtweise, dass oftmals umkämpft ist, worum es in einem Policy-Feld überhaupt geht - auch darum, was nicht legitimer Gegenstand der Auseinandersetzung ist - und wo dementsprechend die Grenzen eines Handlungsbereichs verlaufen.

Es stellt sich die empirische Frage, ob, wo und wie das Phänomen unscharf begrenzter, überlappender oder dezentrierter Policy-Felder auftritt. Welche PolicyFelder sind stärker ausdifferenziert und definieren einen Handlungsbereich mit klaren Grenzen - welche Felder sind diffuser, weniger klar definiert umgrenzt und dezentriert? Ein besonderes Augenmerk sollte dabei jenen Policy-Feldern gelten, für die von Akteuren ein Querschnittscharakter beansprucht wird. Wenn es eine Situation unklar definierter und umkämpfter Grenzen gibt, so wird man nicht von einer statischen Situation ausgehen können. Wie verändern sich Grenzziehungen und Felddefinitionen im Zeitverlauf? Dies sind wohlgemerkt Fragen deskriptiver Art. Bevor der Eindruck der Begrenztheit des Begriffs des Politikfelds eine theoretische Neufassung dieser für die Policy-Analyse grundlegenden Kategorie veranlasst, sollte erfasst und vermessen werden, wie weit verbreitet dieses Phänomen eigentlich ist. Angestrebt ist ein systematischer, über Einzelfallstudien hinausgehender Beleg, dass „unscharfe Grenzen“ (Reckwitz 2008) von Politikbereichen mehr sind als eine bloß punktuell auftretende, mithin vernachlässigbare Anomalie.

Im Hinblick auf eine Operationalisierung von Querschnittsförmigkeit haben aktuelle empirische Arbeiten Vorschläge unterbreitet, wie Konzentration und Dezentrierung von Policy-Feldern mittels geeigneter Konzentrationsmaße gemessen werden können (May u. a. 2005, May u. a. 2006, May u. a. 2011). ${ }^{3}$ Dabei wird aber von einem durch die Forscher vordefinierten Set von Policy-Feldern ausgegangen. Dies schränkt die Erkennbarkeit neu entstehender Felder ein. Derartige Restriktionen sind bislang vor allem erforderlich, um die Materialfluten zu lichten, die bei der Beschäftigung mit Policy-Feldern mit unklar definierten Grenzen zu bewältigen sind. Hier soll korpusunterstützte Politikforschung als stärker explorativer Forschungsansatz eingeführt werden, welcher bei der Analyse der diskursiven Konstruktion von Policy-Feldern neue Möglichkeiten eröffnet. Korpusunterstützte Po-

3 Von diesen Arbeiten zu ,boundary-spanning policy regimes“ zu unterscheiden sind Forschungsansätze, welche Interdependenzen zwischen Politikbereichen untersuchen (Bönker 2008; Jones/ Jenkins-Smith 2009). Letztere gehen davon aus, dass vom Fortbestehen einer grundsätzlichen Differenzierungsstruktur ausgegangen werden kann - eine Annahme, von der hier angesichts des Wandels bestehender und der Genese neuer Felder nicht ausgegangen wird. 
litikforschung ist verwandt mit der computerunterstützten Textanalyse (vgl. Züll/ Mohler 1992; Brier/Hopp 2010), welche durch den Einsatz von Korpora - großen Sammlungen maschinell verarbeitbaren Texts - spezifische Möglichkeiten gewinnt (Bubenhofer 2009). Die Datengrundlage der folgenden Untersuchung ist dabei ein „PolMine“-Plenardebattenkorpus, das alle Debatten im nordrhein-westfälischen Landtag der 14. und 15. Wahlperiode (2005 bis 2012) umfasst. ${ }^{4}$ Das Ziel dieses Aufsatzes ist es, auf dieser Datengrundlage das Ausmaß der diskursiven Streuung von Policy-Feldern zu bestimmen und Folgerungen für die weitere Forschung zu grenzüberschreitenden Policy-Feldern zu ziehen. Nicht zuletzt soll das Potenzial korpusunterstützter Forschung gezeigt werden.

Als Indikator für einen Sinnbezug zu einem Policy-Feld wird hier das Konzept des „Feldmarkers“ verwendet. Dieses wird im folgenden Abschnitt zunächst eingeführt. Durch eine Kombination eines korpusgetriebenen Verfahrens mit einer Auswertung von Standardwerken der Policy-Analyse wird eine Liste mit 24 PolicyFeldern begründet, die im Folgenden untersucht werden. Diese Felder werden zunächst anhand eines Hirschman-Herfindahl-Index (HHI) synchron vergleichend untersucht. Danach gilt das Interesse Trends zur Konzentration und dem Phänomen der Fluktuation - dem Wandern der politischen Aktivitätszentren bei dezentrierten Feldern. Abschließend soll argumentiert und illustriert werden, dass korpusunterstützte Forschung insbesondere eine Exploration großer Mengen von Textdaten ermöglicht, die zwar quantifizierend ist, aber stets den Weg „zurück zum Text“ offen hält.

\section{Policy-Felder und Bedeutungszuweisung durch Feldmarker}

Die hier verfolgte Zielsetzung ist, den Grad der Ausdifferenzierung von PolicyFeldern mit einem geeigneten Maß zu bestimmen und somit Policy-Felder in einem Kontinuum zwischen Ausdifferenzierung und Dezentrierung einzuordnen. Grundlage des hierfür verwendeten Hirschman-Herfindahl-Index (vgl. 3.b) ist eine Zählung der Häufigkeiten sogenannter „Feldmarker“ in Subkorpora des Plenardebattenkorpus, wobei die Subkorpora entsprechend einer von der nordrhein-westfälischen Landtagsverwaltung verwendeten Klassifikation von Debatten nach Sachbereichen gebildet werden (vgl. 3.c). Zunächst ist aber zu begründen, wie das Konzept der Feldmarker theoretisch begründbar ist (2.a) und welche Policy-Felder Gegenstand der weiteren Untersuchung sind (2.b).

4 Informationen zum Plenardebattenkorpus finden sich auf der Website des PolMine-Projekts www.polmine.de. Dort finden sich in einer Dokumentation auch insbesondere Erläuterungen, mit welchen Verfahren das Korpus aufbereitet wurde (Blätte/Berenz 2012). 


\section{a) Die Herstellung von Feldbezügen durch Feldmarker}

Die diskursive Verortung eines Programms im Kontext eines Feldes kann als Aktivierung eines Frames verstanden werden. Analysen von Frames und FramingProzessen betonen die Möglichkeit alternativer Interpretationsschemata (Benford/ Snow 2000; Scheufele 2003), die Untersuchung zur Zentrierung und Dezentrierung von Policy-Feldern findet konzeptionell beim Frame-Konzept einen geeigneten Anknüpfungspunkt. Als operationale Definition des Konzepts der Frames wird im Folgenden davon ausgegangen, dass im politischen Sprachgebrauch bei der Diskussion einer politischen Materie der Bezug zu einem Feld über spezifische Schlagwörter hergestellt wird, die ich als „Feldmarker“ bezeichne. Konkret handelt es sich dabei um solche Wortschöpfungen, die auf ,politik“ enden, etwa „Wirtschaftspolitik“, „Umweltpolitik“ oder „Innovationspolitik“. Feldmarker sind im linguistischen Sinne Schlagwörter, indem sie komplexe Sachverhalte und Zusammenhänge kondensieren (Schröter/Carius 2009: 20). Bezogen auf die sozialwissenschaftliche Feldtheorie sind Feldmarker Wörter, die markieren, worum es bei der Diskussion eines Gegenstands überhaupt geht (Fligstein/McAdam 2012), welchem Feld ein Debattengegenstand zuzurechnen ist, welche Bedeutungen, Akteure und Institutionen überhaupt einschlägig sind. In diesem Sinne treten Feldmarker in Formulierungen auf wie „Die Förderung sozialen Wohnraums ist also Sozialpolitik“ (Heinz Sahnen [CDU], NW-PIPr 14/87), „Schuldner- und Insolvenzberatung ist ein effektives Instrument der Sozialpolitik“ (Armin Laschet [CDU], NW-PIPr 14/142) oder auch in negativen Formulierungen wie „Das ist keine vorsorgende Umweltpolitik“ (André Stinka [SPD], NW-PIPr 14/37). Eine Vielzahl weiterer Belege ließe sich anführen. Entsprechend der These, dass Akteure durch ihre sprachlichen Äußerungen in einem als Framing zu verstehenden Prozess den Bezug einer Beratung zu einem Feld über Feldmarker herstellen, sind Feldmarker der Gegenstand der Frequenzzählungen, welche Basis der Messung von Konzentration und Streuung sind. ${ }^{5}$

Es ist natürlich stark vereinfachend, davon auszugehen, dass Feldmarker als Schlagwörter den Sinnbezug zu einem Feld herstellen und so den Bedeutungskontext einer Debatte definieren. Die folgenden Erwägungen mögen diese These plausibilisieren. Ein erster Einwand wäre, dass die Messung von Sinnbezügen mit Frequenzzählungen von Feldmarkern nicht valide sein könne, weil Bezugnahmen oft implizit hergestellt würden. Dieses Argument kann etwa aus George Lakoffs Konzept der „deep frames“ abgeleitet werden, demzufolge ein oberflächlicher, chan-

5 Die Begriffsbildung der „Feldmarker“ ist nicht zu verwechseln mit Diskursmarkern, die eine spezifische linguistische Bedeutung haben. 
gierender Sprachgebrauch und eine tieferliegende Metaphorik zu unterscheiden sind (Lakoff/Wehling 2008). Dieser Einwand ist nicht grundsätzlich von der Hand zu weisen. Doch im Falle von Feldmarkern werden mit den Begriffen Feldbezüge explizit gemacht, um strittige Territorien zu reklamieren oder um zu sagen, worum es gerade nicht geht. Politische Sprache zeichnet sich durch permanente Wiederholungen aus, so dass strittige Begriffe besetzt und undeutliche Sinnbezüge geklärt werden. ${ }^{6}$ Tatsächlich ist die Zählung von Worthäufigkeiten ein in der Politikwissenschaft gängiges Mittel, um politisch relevante Bezugnahmen zu identifizieren. In einer auf parlamentarische Materialen bezogenen Studie zur Konjunktur des Themas der Generationengerechtigkeit untersucht etwa Jörg Tremmel (2011) die Frequenzen des Wortes „Generationengerechtigkeit" und verwandter Begriffe in Protokollen des Bundestages und in Bundestagsdrucksachen. Frequenzbasierte Inhaltsanalysen sind aber gerade in der Forschung zu Parteipositionen bedeutsam geworden: Der Salienztheorie des Wahlverhaltens zufolge orientieren sich Wähler an den ideologischen Positionierungen der Parteien und den damit verbundenen Schwerpunkt- und Wertsetzungen (Roberston 1976; vgl. Pappi/Shikano 2004). Parteien wiederum markieren ihren Standpunkt über eine issue saliency. Praktisch wird dies auch durch eine Frequenzzählung der Wörter realisiert, mit denen eine bedeutsame Wertsetzung verbunden ist. ${ }^{7}$

Die These der Herstellung von Feldbezügen über Feldmarker und das daran anknüpfende wörterbuchbasierte Zählverfahren teilen die Annahme dieser Ansätze, dass die Frequenzen bestimmter Wörter mit einer Zuweisung von Bedeutung und politischem Gewicht korrelieren. Eine Operationalisierung des Konzepts des Feldmarkers kann zweifelsfrei noch komplexer sein, als nur Wortbildungen auf ,,-politik" zu erfassen: Feldbezüge werden auch dann hergestellt, wenn ein debattiertes Issue als ,integrationspolitische Frage“ oder als Frage der „Integration“ eingeordnet wird, oder indem eine gesetzliche Maßnahme als Antwort auf den „Klimawandel“ dargestellt wird. Bei der Bezeichnung eines Gesetzes als „Integrationsgesetz“ oder eines Programmes als „Klimaschutzprogramm“ trägt die Bezeichnung eines Maßnahmenbündels bereits die Markierung eines Feldes in sich. Für das Konzept des

6 Ironie und uneigentliches Sprechen ist wegen der Gefahr von Missverständnissen ein in der Politik seltenes Stilmittel. Politische Sprache ist voller Anspielungen, aber wo Begriffe umkämpft sind, werden diese gebraucht und möglichst besetzt.

7 Das hier verfolgte Verfahren unterscheidet sich durch die inhaltliche Begründung der Lexeme, die in die Frequenzzählung einbezogen werden von den mittlerweile bei der Vermessung von Parteipositionen populären Wordscore- bzw. Wordfish-Verfahren (Laver/Benoit/Garry 2003; Slapin/ Proksch 2008). Bei diesen Verfahren erfolgt keine theoretische Begründung, welche Wörter in die Analyse einbezogen werden. Es handelt sich um reine Textstatistik ohne Annahmen über die mögliche Bedeutung einzelner Wörter. 
Feldmarkers können sehr komplexe sprachbasierte Messverfahren gefunden werden. Das Plenardebattenkorpus bietet in der Tat eine Grundlage für korpus- und computerlinguistische Auswertungsverfahren, die eine automatisierte Identifizierung deutlich komplexer operationalisierter Frames ermöglichen. Für eine erste vergleichende, korpusunterstützte Untersuchung des Grades der Konzentration von Policy-Feldern und des Problems der Grenzüberschreitungen erscheint das hier gewählte Messkonzept vorerst hinreichend valide.

\section{b) Feldmarker in nordrhein-westfälischen Plenardebatten}

Akteure verleihen bei der Auseinandersetzung um konkrete politische Maßnahmen ihrem Handeln durch das Bewusstsein und die Interpretation, in einem bestimmten, als solchen benennbaren sozialen Zusammenhang - einem Policy-Feld - zu agieren, Bedeutung. Politikwissenschaftler untersuchen, meist ausgehend von solchen Konstruktionen erster Ordnung, die so konstruierten Zusammenhänge politischen Handelns als Politikfelder bzw. Policy-Felder. Dabei handelt es sich dann um Konstruktionen zweiter Ordnung. Im Folgenden soll durch eine Kombination beider Perspektiven eine Liste jener Felder gewonnen werden, die dann näher untersucht werden. Die Klassifikation der Politikfelder deutscher Landesregierungen, die von Pappi, Schmitt und Linhart (2008) als Vorarbeit zu einer Theorie der Koalitionsverhandlungen erstellt wurde zeigt, dass es sich dabei um ein in keiner Weise triviales Problem handelt.

Eine Suche im Volltext aller Plenardebatten im nordrhein-westfälischen Landtag der 14. und 15. Wahlperiode $(2005-2012)^{8}$ nach allen Wörtern, die auf ,politik“ enden, ergibt zunächst eine Liste, die mehr als 600 derartige Komposita umfasst. Als Feldmarker können allerdings nur die Wortbildungen gelten, die einen Feldbezug herstellen. Auf ,politik“ endende Komposita sind oftmals kreative, selten vorkommende Wortschöpfungen, die eingesetzt werden, um das Handeln des politischen Gegners abzuwerten: „Schlafmützenpolitik“, „Kiespolitik“, „Schreibtischpolitik“, „Luftblasenpolitik“, „Schnellschusspolitik“, „Scheuklappenpolitik“ oder „Sprechblasenpolitik“ mögen hier als Beispiele genügen. Öfter gebrauchte Wörter zur Brandmarkung eines politischen Stils oder Ansatzes sind etwa „Symbolpolitik“, „Verschuldungspolitik“ oder „Klientelpolitik“. Jene Wortbildungen sind inhaltlich gesehen keine Feldmarker und müssen nicht weiter berücksichtig werden. Dies gilt allerdings auch für Bezeichnungen für das Handeln auf einer Ebene des europäi-

8 Eine technische Einführung in das Plenardebattenkorpus erfolgt in Abschnitt 3, weil erst dann ein technisches Verständnis des Korpus erforderlich wird. 
schen Mehrebenensystems. Kommunal-, Landes-, Bundes- und Europapolitik wurden aus diesem Grund ebenfalls nicht weiter berücksichtigt.

Im Sinne eines Abgleichs der Konstruktionen ,erster Ordnung“ mit jenen „zweiter Ordnung" kann man eine solchermaßen grundbereinigte Liste mit einer Liste vergleichen, welche auf der Grundlage der Beiträge politikwissenschaftlicher Überblickswerke gebildet wurde, die eine Übersicht über verschiedene Policy-Felder geben. Hierfür wurden zwei Sammelbände zu Policy-Feldern auf Landesebene herangezogen (Schneider/Wehling 2006; Hildebrandt/Wolf 2009) sowie drei auf die Bundesebene bezogene Bände (Schmidt/Zohlnhöfer 2006; Egle/Zohlnhöfer 2007; Egle/Zohlnhöfer 2010). ${ }^{9}$

Gleicht man die grundbereinigte Feldmarker-Liste mit der politikwissenschaftlichen Doxographie ab, so zeigt sich, dass ein eher neues Policy-Feld wie die Klimapolitik in jenen politikwissenschaftlichen Bänden noch nicht behandelt wird, während es in Debatten oft referenziert wird. Es ergeben sich außerdem Hinweise, dass Felder wie die Sport- und Innenpolitik beim Policy-Feld-Vergleich zu beachten sind. Tabelle 1 listet als Ergebnis in der Reihenfolge ihrer Häufigkeit die im Korpus vorgefundenen, auf,,politik“" endenden Komposita auf, die inhaltlich als Feldmarker qualifiziert werden können ${ }^{10}$ und gibt an, in welchen politikwissenschaftlichen Sammelbänden die entsprechenden Policy-Felder behandelt werden. Die letzten drei Spalten geben die absoluten Häufigkeiten der Feldmarker in Debatten der 14. und 15. Wahlperiode sowie im Korpus insgesamt an. Die Tabelle präsentiert somit das Ergebnis des Abgleichs von politikwissenschaftlicher Literatur und Korpusauswertung. Dabei ist die im Folgenden verwendete Feldmarker-Liste allerdings nicht die Schnittmenge jener beiden Listen. Aufgenommen wurden vielmehr nur solche Marker, die pro Jahr mindestens zweimal auftreten. Diese auf Jahre bezogene Frequenzschwelle ergibt sich aus der Zielsetzung, die Entwicklung der Konzentration von Policy-Feldern korpusunterstützt im Zeitverlauf zu untersuchen. Würde

9 Es wäre in weiterführenden Studien lohnenswert, die im politischen und im wissenschaftlichen Sprachgebrauch vorgefundenen Bindestrich-Konstruktionen näher zu untersuchen. Gemeint sind damit Fälle, in denen zwei Wörter in Kombination zur Bezeichnung eines Feldes verwendet werden (z. B. „Migrations- und Integrationspolitik“). In den Überschriften der herangezogenen Sammelbände finden sich insgesamt neun solcher Bindestrich-Konstruktionen. Beim Abgleich mit dem Korpus zeigt sich allerdings, dass die in den politikwissenschaftlichen Aufsätzen zugrunde gelegten Verbindungen im politischen Sprachgebrauch - jedenfalls in dem des nordrhein-westfälischen Landtags - nur eine untergeordnete Rolle spielen. Hier erscheint es wegen der insgesamt vergleichsweise niedrigen Frequenzen dieser Bindestrich-Konstruktionen gut vertretbar, die Analyse auf die Feldmarker zu beschränken, die aus einem Wort gebildet sind.

10 Dementsprechend wurden ausgeschlossen: Regierungspolitik, Symbolpolitik, Verschuldungspolitik, Parteipolitik, Landespolitik, Klientelpolitik, Bundespolitik, Geschäftspolitik, Personalpolitik, Sparpolitik, Schuldenpolitik, Kommunalpolitik, Konsolidierungspolitik, Förderpolitik. 
Andreas Blätte

Tabelle 1: Policy-Felder und Feldmarker in Sammelbänden und im Plenardebattenkorpus

\begin{tabular}{|c|c|c|c|c|c|c|c|c|}
\hline & 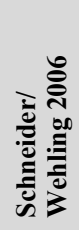 & 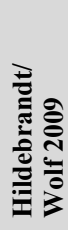 & 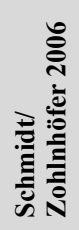 & 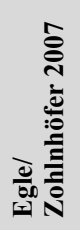 & 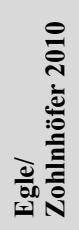 & 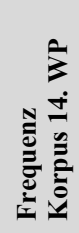 & 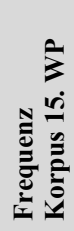 & 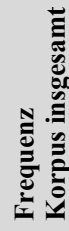 \\
\hline Bildungspolitik & $\mathrm{X}$ & & $\mathrm{X}$ & $\mathrm{X}$ & $\mathrm{X}$ & 656 & 122 & 778 \\
\hline Schulpolitik & & $\mathrm{X}$ & & & & 397 & 171 & 568 \\
\hline Finanzpolitik & & $\mathrm{X}$ & & $\mathrm{X}$ & $\mathrm{X}$ & 327 & 186 & 513 \\
\hline Haushaltspolitik & $\mathrm{X}$ & & & & & 248 & 177 & 425 \\
\hline Wirtschaftspolitik & & $\mathrm{X}$ & $\mathrm{X}$ & & & 296 & 88 & 384 \\
\hline Integrationspolitik & & $\mathrm{X}$ & & & & 233 & 141 & 374 \\
\hline Arbeitsmarktpolitik & & $X$ & & & & 260 & 46 & 306 \\
\hline Umweltpolitik & & & $\mathrm{X}$ & $\mathrm{X}$ & $\mathrm{X}$ & 270 & 35 & 305 \\
\hline Verkehrspolitik & & $\mathrm{X}$ & $\mathrm{X}$ & & & 222 & 57 & 279 \\
\hline Sozialpolitik & & $X$ & $X$ & $\mathrm{X}$ & $\mathrm{X}$ & 202 & 69 & 271 \\
\hline Hochschulpolitik & & $\mathrm{X}$ & & & & 203 & 28 & 231 \\
\hline Klima(schutz)politik & & & & & & 181 & 25 & 206 \\
\hline Medienpolitik & $\mathrm{X}$ & & & & & 187 & 18 & 205 \\
\hline Familienpolitik & & & & & $\mathrm{X}$ & 160 & 20 & 180 \\
\hline Gesundheitspolitik & & & $\mathrm{X}$ & $\mathrm{X}$ & $\mathrm{X}$ & 123 & 43 & 166 \\
\hline Frauenpolitik & & & & & & 137 & 25 & 162 \\
\hline Industriepolitik & & & & & & 86 & 67 & 153 \\
\hline Innovationspolitik & & & & & & 104 & 16 & 134 \\
\hline Agrarpolitik & & & $\mathrm{X}$ & & & 112 & 19 & 131 \\
\hline Strukturpolitik & & & & & & 102 & 32 & 120 \\
\hline Wohnungspolitik & & & $\mathrm{X}$ & & & 86 & 33 & 119 \\
\hline Innenpolitik & & & & & & 94 & 14 & 108 \\
\hline Sportpolitik & & & & & & 46 & 5 & 51 \\
\hline Wissenschaftspolitik & & & & & & 40 & 10 & 50 \\
\hline
\end{tabular}

Eigene Zusammenstellung. 
keine jahresbezogene Frequenzschwelle verwendet oder diese niedriger angesetzt, würden in den Auswertungen bei einem nur einmaligen Auftreten eines Markers inhaltlich nicht aussagekräftige Ausschläge bei Konzentrationswerten auftreten, welche den Aussagewert der Verlaufskurven substantiell in Frage stellen würden.

\section{Konzentration, Überlappung und Dezentrierung von Policy-Feldern}

Die eingangs gestellte Frage nach dem Grad der Ausdifferenzierung oder Dezentrierung von Policy-Feldern läßt sich nach der Diskussion des Konzepts des Feldmarkers spezifizieren: Wie stark konzentriert oder gestreut sind die anhand von Feldmarkern ermittelbaren Bezugnahmen auf Policy-Felder? Wie bei der Operationalisierung der Feld-Frames über Feldmarker sind auch hier komplexere textbasierte Operationalisierungen denkbar. ${ }^{11}$ Der hier realisierte Ansatz besteht darin, Konzentration bzw. Dezentrierung auf der Basis einer Verknüpfung einer sachpolitisch-inhaltlichen Klassifikation von Debatten, die als Zuordnung zu einem PolicyFeld verstanden werden kann, mit den in diesen Texten über Feldmarker erfolgenden Bezugnahmen auf Policy-Felder zu bestimmen. Die Klassifikation von Texten eines Korpus wird dann als Angabe eines primären Feldbezugs verstanden und in Beziehung gesetzt zu den aus dem Text gewonnenen Bezugnahmen auf Felder. Konkret: Die so angelegte Auswertung untersucht, ob in Debatten der Kategorie „Bildung“ nur der Feldmarker „Bildungspolitik“ auftritt, oder ob etwa mit dem Feldmarker „Integrationspolitik“ ein Bezug zu einem weiteren Policy-Feld hergestellt wird. Dieser Ansatz kann nicht zuletzt deswegen verfolgt werden, weil für die Debatten im nordrhein-westfälischen Landtag eine Klassifikation verfügbar ist, die eine für die Berechnung von Konzentration erforderliche Unterteilung des Korpus in Subkorpora ermöglicht. Zunächst soll also das Plenardebattenkorpus erläutert werden (3.a), dann wird der Hischman-Herfindahl-Index als verwendetes Konzentrationsmaß kurz vorgestellt (3.b) und schließlich die verwendete Klassifikation der Plenardebatten erläutert (3.c).

\section{a) Korpus und Datenbank als Ressourcen}

Die Datengrundlage der nachfolgenden Untersuchung ist ein Plenardebattenkorpus mit den insgesamt 2009 Debatten der 14. und 15. Wahlperiode im Landtag Nord-

11 Diskursive Verschränkungen von Feldern könnten auch rein textbasiert ermittelt werden. Relationen von Feldern würden - ohne Nutzung zusätzlicher Datenquellen - aus einem Korpus extrahiert und analysiert. Dies ist sowohl bei der Gewinnung als auch bei der Auswertung solcher relationalen Daten ein anspruchsvoller analytischer Ansatz. 
rhein-Westfalen. ${ }^{12}$ Das Korpus umfasst 14.967.931 Wörter bzw. „Token“, wie es korpuslinguistisch formuliert wird. Abgesehen von den Randjahren des Korpus gab es 2006 bis 2011 im Jahresdurchschnitt 306 Debatten mit 2,3 Millionen protokollierten Token (vgl. Tab. 2). Durch die vorzeitige Auflösung des nordrhein-westfälischen Landtags im März 2012 ist der Umfang des Korpus für das Jahr 2012 deutlich niedriger. Weil bei nur 32 Debatten die Berechnung von Konzentrationsmaßen für 2012 nicht zu belastbaren Ergebnissen führen würde, wurde bei der Analyse der Zeitreihen dieses Jahr nicht berücksichtigt. Das Korpus insgesamt ist - aus korpusund computerlinguistischer Sicht - ein Korpus mittlerer Größe. ${ }^{13}$ Das Korpus wäre aber ganz sicher bei einer manuellen Datenaufbereitung und -auswertung eine Quelle exorbitanten Zeitaufwands. Sprachtechnologische Verfahren, wie sie in Korpusund Computerlinguistik entwickelt wurden, machen den hier verfolgten Forschungsansatz überhaupt erst möglich, indem sie durch Automatisierung eine zeiteffiziente Aufbereitung und Auswertung von Korpora ermöglichen.

Tabelle 2: Zahl der Debatten und Token im Korpus nach Jahren

\begin{tabular}{|l|c|c|}
\hline & Zahl der Debatten & Zahl der Token \\
\hline 2005 & 138 & 927.716 \\
\hline 2006 & 281 & 2.383 .422 \\
\hline 2007 & 333 & 2.400 .118 \\
\hline 2008 & 324 & 2.091 .586 \\
\hline 2009 & 324 & 2.386 .793 \\
\hline 2010 & 264 & 2.036 .921 \\
\hline 2011 & 313 & 2.505 .233 \\
\hline 2012 & 32 & 234.115 \\
\hline Insgesamt & 2.009 & 14.967 .931 \\
\hline
\end{tabular}

Quelle: Eigene Darstellung.

12 Debatten im Sinne des Plenardebattenkorpus sind Tagesordnungspunkte, zu denen im Landtag eine Aussprache stattgefunden hat. Die Zahl der insgesamt im Landtag behandelten Tagesordnungspunkte ist deutlich höher, da viele Tagesordnungspunkte ohne Aussprache behandelt werden.

13 Gängige Referenzkorpora wie das British National Corpus, das American National Korpus oder das DWDS-Kernkorpus umfassen 100 Millionen Wörter. Das Deutsche Referenzkorpus (DeReKO) umfasst (Stand: Februar 2012) 5,4 Milliarden Wörter (vgl. Lemnitzer/Zinsmeister 2010: 105). 
Die Aufbereitung des Korpus erfolgte automatisiert ${ }^{14}$ und mit der Zielsetzung, automatisierte Auswertungen nach variablen Kriterien möglich zu machen. Die Plenarprotokolle, welche die Grundlage der Korpusaufbereitung sind, enthalten als solche kenntlich gemachte Informationen zu Rednern und deren Parteizugehörigkeiten, sowie zu Tagesordnungspunkten. Zwischenrufe sind in Plenarprotokollen ebenfalls satztechnisch (Einrückung, Kursivsetzung) als solche kenntlich gemacht. Solche Informationen sind im aufbereiteten Korpus in Form von „Tags“ bzw. einer „strukturellen Annotation“ erhalten. Technisch ist dies durch ein XML-basiertes Annotationsschema realisiert, welches eine maschinelle Verarbeitung ermöglicht. ${ }^{15}$

Das verwendete Korpus wird als Ressource flankiert von einer Datenbank, die insbesondere eine Tagesordnungspunkt- und Abgeordnetendatenbank ist. Die abgeordnetenbezogenen Tabellen der Datenbank umfassen unter anderem die Parteizugehörigkeiten von Rednern im Parlament. ${ }^{16}$ Die tagesordnungspunktbezogenen Tabellen enthalten eine Klassifikation der Tagesordnungspunkte nach inhaltlichen Kriterien (s. u.). Die Kombination der beiden Ressourcen ermöglicht es, Frequenzzählungen in Partitionen des Korpus (Teilkorpora) vorzunehmen, die nach variablen Kriterien gebildet werden können. Aus praktischen Gründen wurden die sprecherund tagesordnungspunktbezogenen Informationen der Datenbank in das XML-Datenformat des Plenardebattenkorpus geschrieben. Damit wurde es möglich, eine skriptgesteuerte Abfrage des Plenardebattenkorpus ohne zusätzliche Abfrage der Datenbank zu gestalten. Für die grafischen Darstellungen wurde schließlich R verwendet.

14 Ausgangspunkt der Korpusaufbereitung des Plenardebattenkorpus waren die über die Website des Düsseldorfer Landtags frei zugänglichen Plenarprotokolle im html-Format. Im Rahmen eines Pilotprojekts wurden Skripte (Programmiersprache Perl) entwickelt, die eine automatisierte Umwandlung des Rohmaterials in einen Korpus leisten. Unter einem methodologischen Gesichtspunkt (der Reliabilität) sei darauf hingewiesen, dass eine manuelle Durchsicht des gesamten mit automatisierten Verfahren aufbereiteten Korpus nicht möglich wäre. Es kann nicht bemerkte Konvertierungsfehler geben, die bei Frequenzzählungen zu Messfehlern führen. Allerdings ermöglicht die XML-Annotation eine Überprüfung der Daten auf sogenannte „Wohlgeformtheit“ bzw. „Validität“". Fehler im Datenmaterial können dadurch zwar nicht ausgeschlossen werden, sind aber, wie die (extensive) Durchsicht von Stichproben zeigt, nicht erkennbar.

15 Wie bei dem durch Webseiten bekannterem html steht bei XML einem öffnenden Tag $(,<\operatorname{tag}>$ “) immer ein schließender Tag (, $<\backslash$ tag >") gegenüber. Das für das Plenardebattenkorpus verwendete Annotationsschema ist angelehnt an gängige Standards wie TEI oder XCES. Diese gängigen Standards sind nicht angepasst an die Erfordernisse der Annotation eines Plenarprotokolls. Daher wurde ein gesondertes Annotationsschema entwickelt.

16 In den Plenarprotokollen sind die Partei- bzw. Fraktionsmitgliedschaften von Mitgliedern des Landtags angegeben, nicht aber die von Regierungsmitgliedern. Diese (manuell recherchierten) Information findet sich ergänzend in der Datenbank. 
Korpusanalyse bedeutet keineswegs, durch vollständige Automatisierung gegenüber der Bedeutung der Begriffe in ihrem jeweiligen Kontext ignorant zu werden. Im Gegenteil: Korpusunterstützte Forschung kann jederzeit den Weg zurück zum Text antreten und anhand konkreter Textpassagen der Bedeutung von Sprachgebrauch im jeweiligen Kontext nachgehen. Quantifizierende und qualitative Analyse ergänzen einander als Wege zum Dialog mit den Daten. Technisch realisiert wird dies durch den Einsatz der Corpus Workbench (CWB), die als System zur Korpusverwaltung auf dem PolMine-Server installiert ist. Die CWB ermöglicht unter anderem über Schnittstellen zu anderen Programmiersprachen (wie Python, Perl oder R) ${ }^{17}$ automatisierte Abfragen von Frequenzen, sie bietet aber zugleich die Möglichkeit der schnellen und flexiblen Sichtung von Konkordanzen. Die strukturelle Annotation des Korpus ermöglicht es dabei, eine bestimmte Anzahl von Wörtern, den Satz, den Absatz oder einen vollständigen Debattenbeitrag als Kontext des Ergebnisses einer Suchanfrage in Augenschein zu nehmen. Die im folgenden Text angeführten Belegstellen beruhen auf dieser Möglichkeit.

\section{b) Die Messung von Ausdifferenzierung, Überlappung und Dezentrierung}

Die Kombination des entsprechend aufbereiteten Korpus mit Korpusverwaltungssystem und skriptgesteuerter Korpusabfrage ermöglicht die Zählung der Frequenzen von Feldmarkern in Teilkorpora, die entsprechend einer Klassifikation von Basistexten (hier: Debatten) gebildet werden. Auf der Basis von Tabellen (Term-Dokument-Matrizen), die so für jeden Feldmarker gewonnen werden können, lässt sich ein Konzentrationsmaß für jedes Policy-Feld berechnen (hier: Hirschman-Herfindahl-Index). Bevor Klassifikationsschema und Konzentrationsmaß erläutert und diskutiert werden, sei aber die politikwissenschaftliche Intuition hinter diesem als Messinstrument dienendem Konzentrationsmaß anhand dreier idealtypischer Konfigurationen von Policy-Feldern erläutert.

Die Policy-Analyse geht, wie eingangs angesprochen, konventionell vom PolicyFeld mit geklärten Grenzen aus. Begrifflich ist dies der Fall des „Policy-Subsys-

17 In der Linguistik ist die Skriptsprache Perl nach wie vor weit verbreitet. Diese hat den unbestreitbaren Vorteil, dass sogenannte „reguläre Ausdrücke“ integral in die Sprache eingebunden sind. Der Konverter für die Aufbereitung des hier verwendeten Plenardebattenkorpus wurde in Perl geschrieben. Perl hat aber den Ruf einer ,,write only“-Sprache, d.h. den Nachteil, dass Skripte nicht nur für andere, sondern auch für den Programmierer selbst schon nach kurzer Zeit oft nicht mehr nachvollziehbar sind. Python-Skripte sind zuweilen umständlicher, aber im Nachhinein besser nachvollziehbar. Python findet bei der Sprachverarbeitung durch Computerlinguisten oft Anwendung (Bird u. a. 2009) und findet aus dem genannten Grund in wissenschaftlichen Anwendungsbereichen weite Verbreitung (Langtangen 2011). 
tems" bzw. des ausdifferenzierten Politikfelds. Bei dem geschilderten korpusanalytischen Messverfahren würde diesen Fall eine hohe Konzentration der Häufigkeiten von Feldmarkern in einem Subkorpus indizieren, der per Klassifikation aus den Debatten zu einem Policy-Feld gebildet ist. Dem ausdifferenzierten Policy-Feld steht als Gegentypus das dezentrierte Policy-Feld gegenüber. Dies wäre im reinen Fall die politische Realisierung des Anspruchs der Gestaltung von „Querschnittspolitik“, eine Konstellation mit einer quasi ubiquitären Bezugnahme auf ein Querschnittsthema. In dem gemäß Klassifikation in Subkorpora unterteilten Plenardebattenkorpus treten Feldmarker dann nicht konzentriert, sondern mehr oder weniger gestreut über die Subkorpora auf. Zwischen diesen beiden Polen der Typologie liegt der Typus des überlappenden Policy-Feldes: Feldbezüge treten zwar in einem Subkorpus relativ konzentriert auf, sind aber nicht nur ausnahmsweise in anderen Bereichen ebenfalls zu finden. Abbildung 1 illustriert mögliche Messungen bei den so charakterisierten Typen des ausdifferenzierten, überlappenden und dezentrierten Policy-Feldes.

\section{Abbildung 1: Idealtypen von Policy-Feldern}

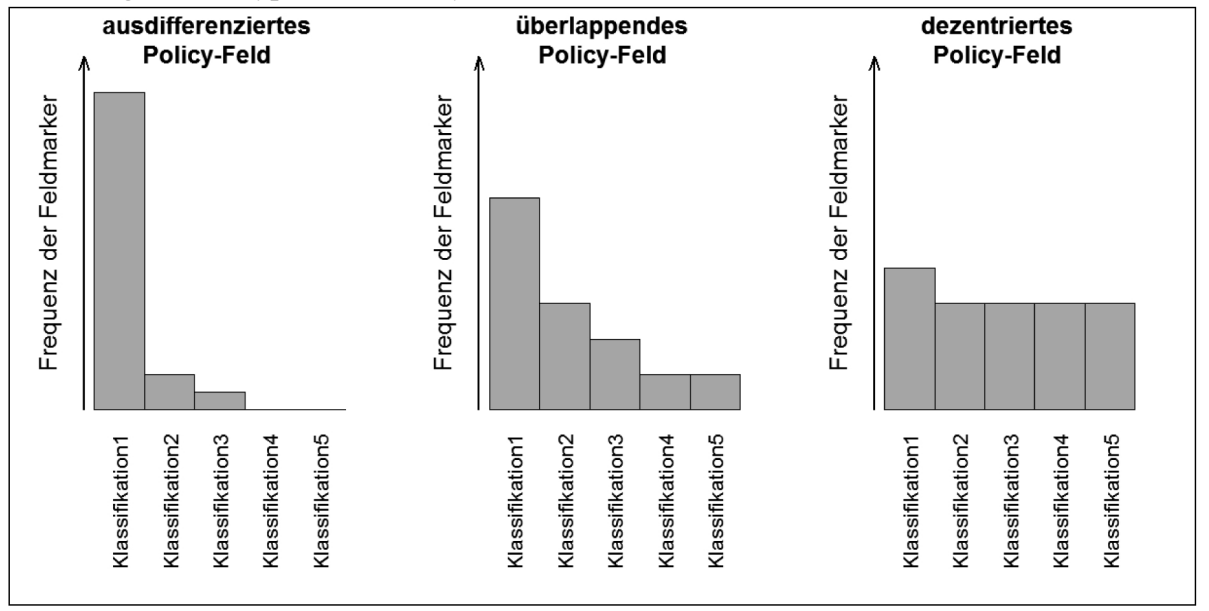

Quelle: Eigene Darstellung.

Diese Typologie hat Parallelen zu einer Unterscheidung von „policy subsystems“ und ,boundary-spanning policy regimes“, die Ashley Jochim und Peter May (2010) 
vorschlagen. ${ }^{18}$ Hier soll allerdings die Annahme überwunden werden, es gebe substantielle Policy-Subsysteme einerseits und mehr oder weniger stabile grenzüberschreitende Policy-Regime andererseits. Dies eröffnet die Möglichkeit genetischer Prozesse - die Entwicklung von dezentrierten zu ausdifferenzierten Feldern. Übernommen wird gleichwohl die von Peter May u. a. (May/Sapotchine/Workman 2006; May/Jochim/Sapotchine 2011) vorgeschlagene Messung der Konzentration mit einem Hirschman-Herfindahl-Index (HHI). Während in der angesprochenen Forschung diese Messung die institutionelle Absicherung eines ,,policy regmines“ über die Präsenz der Vertreter maßgeblicher Behörden in Anhörungen zu Maßnahmen relevanter Policy-Subsysteme erfolgt (May/Jochim/Sapotichne 2011: 299 f.), wird hier die Konzentration diskursiver Bezugnahmen gemessen.

Der HHI wird berechnet als der aufsummierte quadrierte Anteil der Häufigkeit eines Merkmals (hier: Frequenz von Feldmarkern) bei verschiedenen Merkmalsträgern (hier: $n$ Subkorpora) an der insgesamt (im Korpus) feststellbaren Merkmalshäufigkeit (HHI $=\sum \mathrm{a}_{\mathrm{i}}{ }^{2}$ mit $\left.\mathrm{a}_{\mathrm{i}}=\mathrm{tf}_{\mathrm{i}} / \sum \mathrm{tf}\right)$. Die Werte des Hirschman-HerfindahlIndex bewegen sich im Bereich zwischen $1 / \mathrm{n}$ und 1, wobei ein Wert von 1 für eine vollständige Konzentration steht. Je mehr sich der HHI seinem Minimalwert (bei 50 Kategorien $1 / 50$ bzw. 0.02) annähert, desto geringer ist die Konzentration. Der HHI hat zwar sein Haupteinsatzgebiet in wirtschaftswissenschaftlichen Studien zur Marktkonzentration, empfiehlt sich aber gleichwohl im gegebenen Zusammenhang aus Gründen der Anschlussfähigkeit zur vorliegenden Forschung zu grenzüberschreitenden Policies. Es handelt sich um einen transparent interpretierbaren Index. Die für die Berechnung des Index erforderlichen Zahlenwerte können korpusunterstützt gewonnen werden.

\section{c) Zur Klassifikation der Plenardebatten}

Die Berechnung des HHI für Feldmarker setzt eine Tabelle mit den Frequenzen der Feldmarker in Subkorpora voraus. Die Bildung der Subkorpora erfordert eine Klassifikation der parlamentarischen Debatten. Für diese Klassifikation wurde eine Systematik des Landtags Nordrhein-Westfalen mit 163 Kategorien genutzt: Jede Debatte im Düsseldorfer Landtag wird von der Parlamentsverwaltung des Landtags mit Hilfe dieser Kategorien klassifiziert, diese Klassifikation ist über die Website

18 Fruchtbar kann es sein, diese grenzbezogene Typologie in einen Dialog mit Klassifikationen von Policy-Netzwerken zu bringen. Dabei ergibt sich allerdings das Problem, das Netzwerkanalysen aus methodischen Grenzen immer davon ausgehen müssen, dass das ,,boundary specification problem“ gelöst werden kann - eine Annahme, die hier gerade bestritten wird. 
des Landtags öffentlich zugänglich. ${ }^{19}$ Diese Klassifikation wurde (automatisiert) den Tagesordnungspunkten zugewiesen, zunächst in die Datenbank übernommen und dann, um den Auswertungsprozess zu vereinfachen, in das Plenardebattenkorpus übertragen. ${ }^{20}$ Zwei bei der Verwendung der Systematik zu treffende Entscheidungen bedürfen allerdings einer näheren Diskussion. Erstens kann die Systematik des Landtags in ihrer feingliedrigen Variante mit 163 Kategorien nicht zu aussagekräftigen Ergebnissen führen. Die Ausdifferenzierung des Schemas führt zu einer gleichmäßigen Streuung von Häufigkeiten über klein dimensionierte Subkorpora, die kaum Aussagewert hat. Für die Bildung der Subkorpora wurden aus diesem Grund nicht diese Unterkategorien herangezogen, sondern Oberkategorien, welchen entsprechend einem Schema der Verwaltung des Landtags Nordrhein-Westfalen die Unterkategorien zugeordnet werden können. Die Liste jener thematischen Bereiche umfasst 63 Oberkategorien, die Einträge entsprechen weit überwiegend inhaltlichen Zusammenhängen, die als Policy-Felder verstanden werden können. Kategorien allgemein-technischer Art oder die eine Ebene des politischen Systems bezeichnen und die nicht als Policy-Felder gelten können, wurden von der weiteren Untersuchung ausgeschlossen. ${ }^{21}$ Für die Bildung der Subkorpora wurde eine gefilterte Liste mit 50 Kategorien verwendet.

Ein etwas diffizileres Problem entsteht durch Mehrfachklassifikationen. Bei den Sitzungen im Düsseldorfer Landtag während der 14. und 15. Wahlperiode fand zu insgesamt 2009 Tagesordnungspunkten eine Aussprache statt. Zwar reduziert die aggregierte und gefilterte Klassifikation die Zahl der Mehrfachklassifikationen. Immerhin 1.365 Debatten wurden in eindeutiger Weise klassifiziert. Allerdings wur-

19 Neben diesem Klassifikationsschema werden die Tagesordnungspunkte auch verschlagwortet. Die Verschlagwortung folgt aber nicht einem über Zeit hinweg stabilen Schema, sondern basiert auf einer offenen, nicht abgeschlossenen Liste. Dadurch ist die Verschlagwortung der Debatten hier nicht einsetzbar.

20 Die Nutzung eines fremden, primär den Erfordernissen der Parlamentsorganisation entsprechenden, aber nicht nach eigenen wissenschaftlichen Kriterien entwickelten Kategoriensystems, erschien als vertretbar. Zwar ist es im Einzelnen nicht möglich nachzuvollziehen, wie und nach welchen Kriterien die Landtagsverwaltung , ,odiert“" hat, allerdings ist der Effekt, dass eine Klassifikation aus der Binnenperspektive des Parlaments - und nicht durch die wissenschaftliche Beobachterperspektive - erfolgte, durchaus im Einklang mit den hier zugrunde gelegten Annahmen, wie Felder konstituiert werden. Eine Klassifikation nach streng wissenschaftlichen Kriterien (etwa jener des Policy Agendas Project) wäre sicher der Idealfall, doch erwies sich die Klassifikation durch die Landtagsverwaltung bei einer kritischen Durchsicht als stichhaltig genug, um Aussagen zu gewinnen, wie relevant das Phänomen der Konzentration diskursiver Bezugnahmen auf Felder ist.

21 Die Einträge dieser Stoppliste sind: Europäische Union, Staatsaufbau, Nation, Ideologien, Politische Kräfte, Wahlen, Parlament, Öffentliche Verwaltung, Statistik, Verfassungsgerichtsbarkeit, Öffentlicher Haushalt. 
den 505 Tagesordnungspunkte zweifach, 102 dreifach und 28 vierfach klassifiziert. ${ }^{22}$ Allerdings: Mehrfachklassifikationen sind keine Unzulänglichkeit der Arbeitsweise des Düsseldorfer Landtags. Es handelt sich um einen konzeptionell interessanten Beleg für die Problematik grenzüberschreitender Policy-Felder. Immerhin lautet die hier vertretene These, dass die Grenzen zwischen Policy-Feldern weniger eindeutig sind, als es die Begriffe der Politikfelder oder Policy-Subsysteme nahe legen. Wenn aber die Durchdringung von Feldern theoretisch erwartet wird, so bedeutet dies einen Schwund von Eindeutigkeit bei der Beratung von Entscheidungsmaterien: Mehrfachklassifikationen von Debatten sind in der Tat tatsächlich theoretisch zu erwarten; die Mehrfachklassifikationen sind ein Hinweis, wie angebracht es ist, grenzüberschreitenden Policy-Feldern theoretisch und empirisch mehr Aufmerksamkeit zu widmen. Für die Berechnung des Konzentrationsmaßes war das Problem der Mehrfachklassifikationen gleichwohl zu lösen. Es erschien am stringentesten, im Fall von Mehrfachklassifikationen die Häufigkeit des Auftretens eines Feldmarkers (tf für term frequency) in einer $n$-fach klassifizierten Rede jeweils anteilig, das heißt mit dem Wert tf $/ \mathrm{n}$, zu den Zählwerten der einzelnen Kategorien zu addieren. Ausschlaggebend war dabei die Überlegung, dass in der Kontingenztabelle, die in den Zeilen die Feldmarker als Fälle auflistet und in den Spalten die Kategorien, welche die Partition des Korpus angeben, in der ein Feldmarker auftritt, die Zeilensummen der Gesamthäufigkeit des Feldmarkers im Korpus entsprechen sollten.

\section{Konzentration und Dezentrierung bei 24 Policy-Feldern}

Nachdem die Policy-Felder bestimmt sind, die in die Untersuchung einbezogen werden, und erläutert wurde, mit welchem Verfahren diese hinsichtlich ihrer Konzentration verglichen werden, können nun die Ergebnisse der Untersuchung berichtet werden. Zunächst soll dargestellt werden, welche Konzentrationswerte sich ergeben, wenn die Policy-Felder für den gesamten Zeitraum (14. und 15. Wahlperiode) verglichen werden (4.a). Anschließend werden Prozesse der Konzentration im Zeitverlauf untersucht (4.b).

22 Es traten Reden auf, die elf- bis vierzehnfach klassifiziert worden sind (jeweils eine). Dabei handelt es sich um Regierungserklärungen und die zugehörigen Aussprachen. 


\section{a) Konzentration und Streuung: Synchrone Untersuchung}

Betrachtet man nach all diesen Vorüberlegungen die Felder im Vergleich, so bestätigt die Darstellung der nach ihren HHI-Werten in absteigender Reihenfolge sortierten Felder in einem Säulendiagramm, dass es Felder gibt, die einen höheren Konzentrationsgrad aufweisen, und dass dem einige Felder gegenüberstehen, bei welchen die Bezugnahmen auf die zugehörigen Marker stärker streuen. Konzentrationswerte von mindestens 0.5 finden sich bei der Medien-, Schul-, Hochschul-, Sport-, Verkehrs-, Innen-, Integrations- und Wissenschaftspolitik. In diese Gruppe der stärker konzentrierten und ausdifferenzierten Policy-Felder fallen damit gerade auch Bereiche, welche ausgeprägte Kompetenzbereiche der Länder betreffen. Man kann dann eine Zwischengruppe von zehn Feldern mit HHI-Werten von 0.3 bis 0.5

Abbildung 2: Reihung der Felder entsprechend HHI

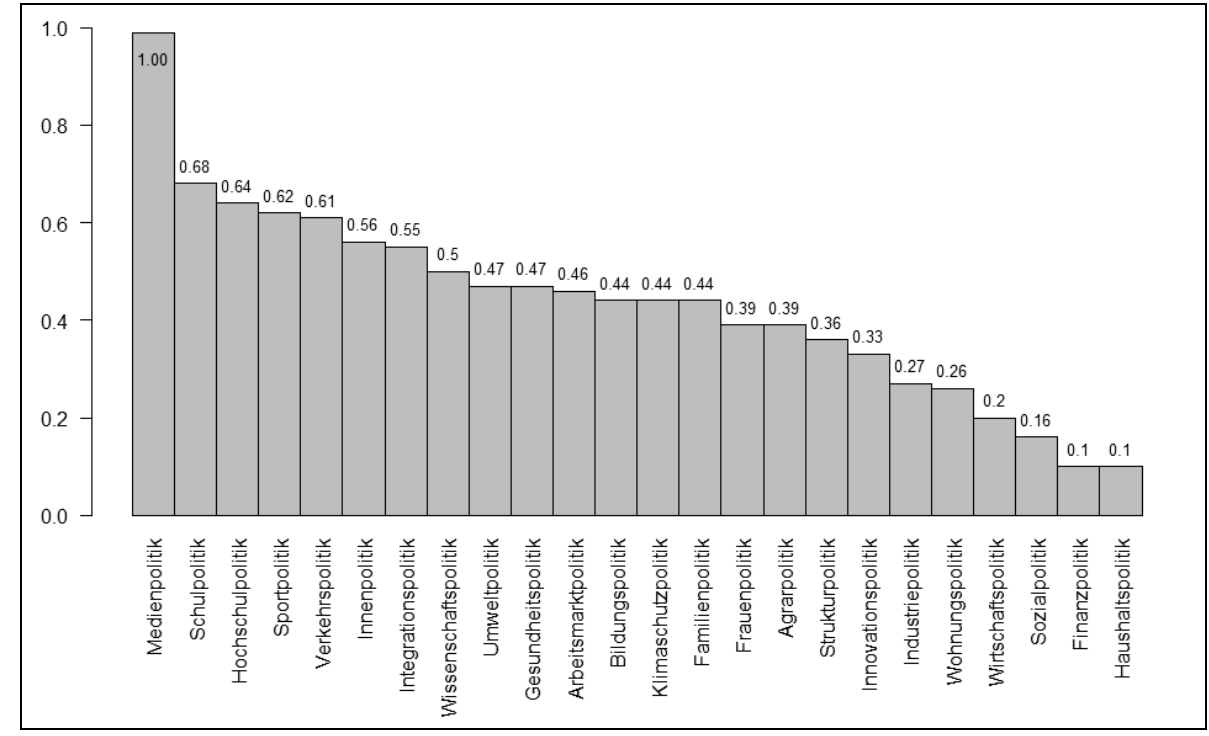

Quelle: Eigene Darstellung. 
identifizieren, bei der Querbezüge und Überlappungen zu anderen Politikbereichen schon deutlich ausgeprägter sind. Mit HHI-Werten unter 0.3 befindet sich am Ende der Reihung eine Gruppe, die als deutlich stärker dezentriert gelten kann. Tatsächlich sind es „klassische“ Politikbereiche wie Wirtschafts- und Sozialpolitik und schließlich Finanz- und Haushaltspolitik, die das Ende der Reihung der Felder entsprechend ihrer HHI-Werte markieren. ${ }^{23}$

Bemerkenswert ist nun vor allem, dass jene Felder, denen Querschnittscharakter zugeschrieben wird, sich entgegen dieser Forderung gerade nicht in der Gruppe der dezentrierten Felder wiederfinden. Mit der Rede vom „Querschnittscharakter“ eines Policy-Feldes ist gemeint, dass ein sektoralisierter Ansatz verfehlt wäre; dass vielmehr die Erfordernisse von Querschnitts-Policies in einer Reihe von Policy-Feldern aktiv politische Maßnahmen erfordern oder zumindest berücksichtigt werden müssen. Entsprechende Formulierungen und Forderungen findet man im Korpus bezogen auf Frauen-,${ }^{24}$ Integrations-, ${ }^{25}$ Familien- ${ }^{26}$ Klima- ${ }^{27}$ und Innovationspolitik. ${ }^{28}$ Wenn sich die Politikbereiche, denen mit größerer Regelmäßigkeit Quer-

23 Man könnte spekulieren, dass die herausgehobene Bedeutung dieser Bereiche eine höhere Streuung von Bezugnahmen auf diese Felder hervorruft. Doch dann müssten auch die Felder im kultuspolitischen Kompetenzbereich geringe Konzentrationswerte aufweisen. Sozial-, Wirtschafts-, Haushalts- und Finanzpolitik unterscheiden sich aber hinsichtlich ihrer Konzentration von jenen landespolitischen Gestaltungsbereichen.

24 So weist Barbara Steffens (Grüne) auf die Formulierung im Koalitionsvertrag von CDU und FDP hin: „Die Gleichstellung von Mann und Frau berücksichtigen wir als Querschnittsaufgabe bei allen politischen Entscheidungen." (PIPr 14/4). Maria Westerhorstmann (CDU) betont dementsprechend: „Frauenpolitik ist heute mehr denn je eine Querschnittsaufgabe, sodass wir an den verschiedensten Stellen ansetzen müssen." (PIPr 14/49).

25 So betont Christian Lindner (FDP), ,[...] dass Integration eine Querschnittsaufgabe ist. Davon sind alle gesellschaftlichen Bereiche betroffen: Schule, Kindergarten, Arbeitsmarkt, Gesundheitswesen, Städtebau.“ (PIPr 14/40) Michael Breuer (CDU) bestätigt: „Wir begreifen Integration als eine Querschnittsaufgabe der Landesregierung.“ (PIPr 14/46).

26 Wie es Ralf Witzel (FDP) formuliert: „Nachhaltige Familienpolitik ist damit nun eine echte Querschnittsaufgabe, die Zukunftschancen sichert. Familienpolitik ist eben nicht nur eine Aufgabe des Bundes, des Landes und der Kommune, sondern die gesellschaftliche Querschnittsaufgabe unserer Zeit.“(PIPr 14/22).

27 Unter Bezug auf den Klimaschutzbericht der Landesregierung hebt Svenja Schulze (SPD) hervor: „Klimaschutz ist eine Querschnittsaufgabe, die viele Politikbereiche wie die Energie-, Verkehrs-, Wirtschafts-, Wohnungsbau-, Umwelt- und Forschungspolitik umfasst. Klimaschutzpolitik ist deshalb integraler Bestandteil des politischen Gesamtkonzepts der Landesregierung, durchgeführt von allen Ressorts.“ Oder in einer Formulierung von Norbert Römer (SPD): „Klimaveränderungen wirken in den verschiedensten Lebensbereichen. Klimaschutzpolitik ist deshalb ein Querschnittsthema." (PIPr 14/82).

28 So Michael Groschek (SPD): „Für uns ist Innovationspolitik Querschnittsaufgabe. Wir müssen Bildung und Betreuung ausbauen, um Begabungsreserven zu heben. Wir brauchen eine bedarfsgerechte U3-Betreuung. Wir brauchen beitragsfreie Kindertagesstätten. Wir brauchen eine Schule, in der länger gemeinsam gelernt wird." (PIPr 14/46). 
schnittscharakter zugeschrieben wird, nicht unter den stark konzentrierten und ausdifferenzierten Policy-Feldern - und vor allem nicht unter den am stärksten dezentrierten Policy-Feldern - befinden, so verdeutlicht dies den primär deklarativen Charakter der Betonung des „Querschnittscharakters“ eines Themas. Eine solche Forderung bedeutet nicht, dass ein diskursives „Sollen“ tatsächlich in ein diskursives ,Sein“ übersetzt wird. Wenn die Fachpolitiker eines Feldes fordern, ihr Anliegen möge in anderen Handlungsbereichen mit berücksichtigt werden, so muss dies keineswegs umgesetzt werden.

Die Forderung, ein Thema als Querschnittsthema zu begreifen, bedeutet aber, dass ein diskursiver Wandel, sozusagen ein Einsickern eines Feldbezuges in weitere Felder, politisch für erforderlich gehalten wird. Haben sich derartige Veränderungen ergeben? Es ist ein wesentliches Ziel bei der Entwicklung des Plenardebattenkorpus, Veränderungen der Konzentration nachgehen zu können. Bei den Querschnittsthemen ist eine „Verquerschnittlichung“ bzw. eine abnehmende Konzentration erst noch zu erwirken. Eine entsprechende Analyse von Zeitreihen ist Gegenstand des folgenden Abschnitts.

\section{b) Konzentrationsprozesse und Zeitreihen}

Jedes Plenarprotokoll nimmt eine zeitliche Einordnung vor: Wahlperiode, Nummer der Sitzung und vor allem das Datum des Sitzungstermins sind angegeben. Plenarprotokolle sind damit eine mögliche Grundlage für Zeitreihendaten. Wie haben sich die Konfigurationen von Policy-Feldern im Laufe der Zeit verändert? Sind Prozesse der Konzentration oder Dezentrierung feststellbar? Diese Frage stellt sich insbesondere bei Politikbereichen, bei denen mit einer Definition als Querschnittsthema eine sinkende Konzentration gefordert wurde oder die einem starken organisatorischen oder konzeptionellen Wandel unterworfen waren. Dies kann auch miteinander verbunden sein: Nordrhein-Westfalen übernahm in der 14. Wahlperiode (2005-2009) in der Integrationspolitik eine Vorreiterrolle, indem in diesem Bundesland das erste Integrationsministerium gegründet wurde. Welche Folgen dies für die Aufmerksamkeit für Fragen der „Integrationspolitik“ hatte, ist allerdings nicht ohne weiteres klar. In der Policy-Analyse würde man diese organisatorische Verfestigung als Triebfeder der Ausdifferenzierung des Policy-Feldes sehen und auch im parlamentarischen Geschehen eine stärkere Konzentration integrationspolitischer Bezugnahmen erwarten: Für gewöhnlich bildet das Parlament die Arbeitsteilung innerhalb der Regierung ab. Andererseits wurde „Integrationspolitik“ weiterhin als Politikbereich mit Querschnittscharakter definiert. Dies spricht für eine gleichbleibend hohe Streuung integrationspolitischer Bezüge. Ein zweites Policy- 
Feld von Interesse ist die Klimapolitik, die sich als politischer Handlungsbereich herausbildet. Auch hier stellt sich die Frage, ob sich eine Sektoralisierung der Klimapolitik vollzieht, oder ob sich ein Feld mit Querschnittscharakter herausbildet.

Abbildung 3: Trends der Dezentrierung und Konzentration im Zeitverlauf

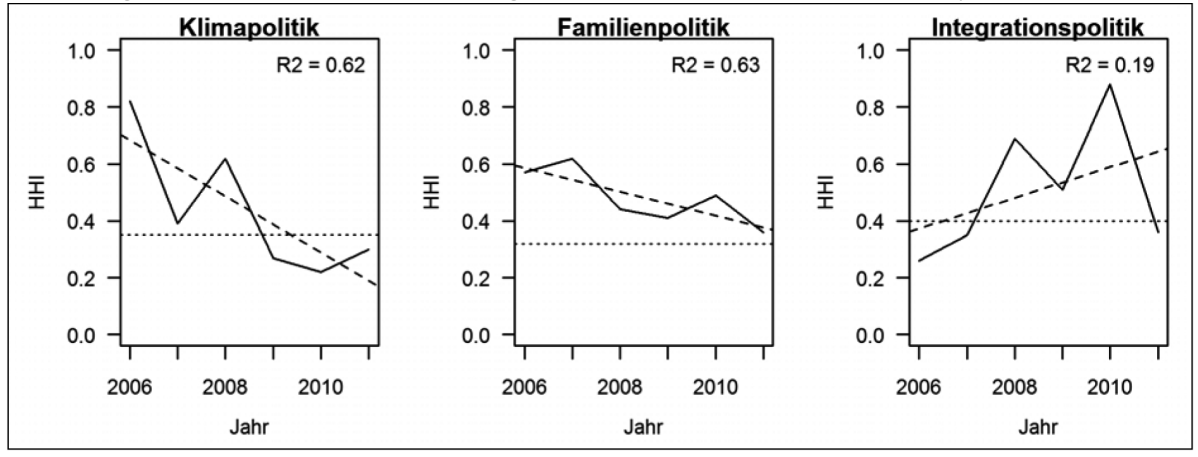

Quelle: Eigene Darstellung.

Ergänzend zu diesen inhaltlichen Erwägungen, die das Interesse auf bestimmte Policy-Felder lenkt, richtet eine Trendanalyse auf Grundlage der aus dem Plenardebattenkorpus gewonnenen Zeitreihendaten die Aufmerksamkeit auch auf solche Policy-Felder, bei denen sich nicht erwartete Konzentrations- oder Dezentrierungsprozesse vollziehen. Eine (lineare) Trendfunktionen lässt sich allerdings für die 24 Policy-Felder nur in zwei Fällen - im Fall der Klimapolitik sowie der Familienpolitik - hinreichend gut schätzen $\left(\mathrm{R}^{2}>0.6\right)$. Für diese beiden Policy-Felder zeigt sich ein Trend fallender HHI-Werte, mithin eine Tendenz zur Dezentrierung. Für die Integrationspolitik lässt sich zwar dem Augenschein nach bis 2010 ein Konzentrationstrend erkennen. Eine dann wieder stark abfallender HHI-Wert nimmt dem Trend dann aber die Eindeutigkeit $\left(\mathrm{R}^{2}=0.19\right) .{ }^{29}$

Eine Annäherung an eine inhaltliche Interpretation des zumindest im Fall der Klima- und der Familienpolitik erkennbaren Dezentrierungstrends ergibt ein Blick auf „Blasendiagramme“, welche nach Jahren unterteilt die Häufigkeit von Feldmarkern in Subkorpora des Plenardebattenkorpus darstellen, die entsprechend den Kategorisierungen des Landtags gebildet wurden (Abb. 4). Diese Diagramme basieren auf entsprechenden Tabellen; die Visualisierung bietet in erster Linie den Vorteil, dass die Daten schneller erfassbar werden, wo bei der Tabelle viele Null-

29 Insgesamt muss man sicher den Versuch der Trendanalyse unter den Vorbehalt stellen, dass für aussagekräftige Ergebnisse eine noch breitere Datenbasis in die Untersuchung einbezogen werden müsste. Ein größerer zeitlicher Umfang des Korpus würde die Ergebnisse belastbarer machen. 
werte den Blick auf die inhaltlich interessanten Werte verstellen würden. Die Diagramme wurden so aufbereitet, dass die vertikale Achse die entsprechend der Kategorisierung der Landtagsverwaltung gebildeten Subkorpora zeigt, die horizontale Achse gliedert diese entsprechend dem Zeitverlauf. Die Fläche der Blasen ist proportional zu der Häufigkeit eines Feldmarkers in einem nach Kategorisierung und Jahr gebildeten Teilkorpus. ${ }^{30}$ Insgesamt wurde die Darstellung auf die acht Subkorpora mit den höchsten Häufigkeiten beschränkt. ${ }^{31}$ Die Zielsetzung dieser Darstellungsform ist, schnell erfassbar zu machen, in welcher Weise Bezugnahmen auf ein Feld von einem (unten angeordneten) Schwerpunkt solcher Bezugnahmen ausstrahlen.

Abbildung 4: Häufigkeiten der Feldmarker „,Klimapolitik“ und „,Familienpolitik“ in Subkorpora

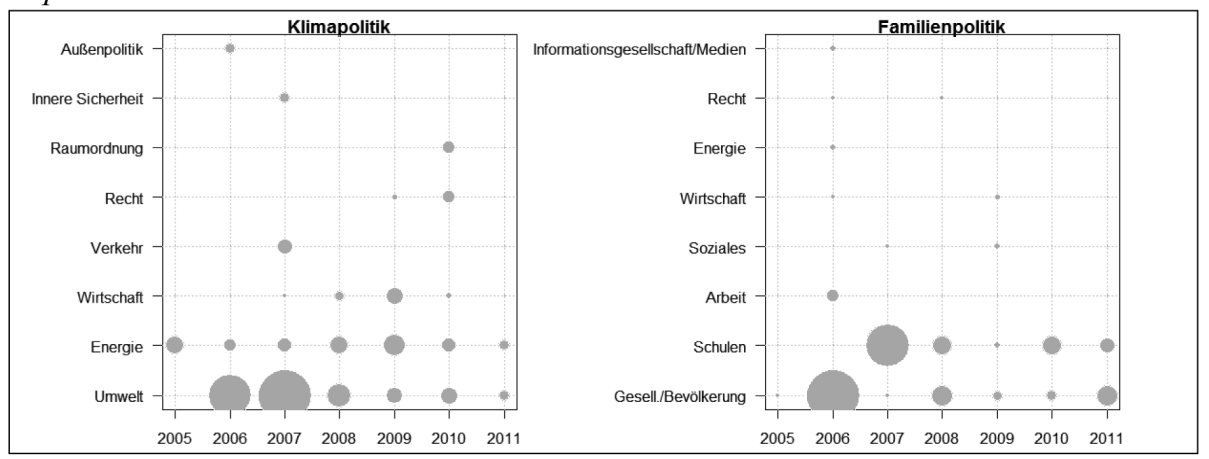

Quelle: Eigene Darstellung; Erläuterung im Text.

Die Trendanalyse zeigte im Falle der Klima- und der Familienpolitik mit einem Trend zu sinkenden HHI-Werten, dass ein Feldbezug in zunehmend weiteren Bereichen politischen Handelns hergestellt wird. Das Blasendiagramm verdeutlicht, was auch die HHI-Werte zum Ausdruck bringen. Im Fall der Klimapolitik sieht man bis 2008 noch eine höhere Konzentration entsprechender Bezugnahmen in den De-

30 Der Radius der Blasen wurde dabei so skaliert, dass die „Blase“, welche die größte Häufigkeit repräsentiert, den Durchmesser 1 erhält. Damit werden Überschneidungen vermieden, welche die Lesbarkeit des Diagramms beeinträchtigen würden.

31 Auf der vertikalen Achse wurden die Kategorien so gruppiert, dass sich die Kategorie / der Subkorpus, in welcher der Feldmarker („Klimapolitik“ / „Familienpolitik“) im gesamten Untersuchungszeitraum am häufigsten auftritt, unten befindet. „Klimapolitik“ tritt etwa am häufigsten in den Debatten der Kategorie „Umwelt“ auf, weshalb sich diese Kategorie im „Klimapolitik“-Blasendiagramm unten befindet. Je weiter oben eine Kategorie angeordnet ist, desto niedriger ist die Gesamthäufigkeit des Feldmarkers im entsprechenden Subkorpus. 
batten der „Umwelt“-Kategorie. In den folgenden Jahren ist diese Konzentration deutlich niedriger. In 2010 etwa kommt es zu solchen Bezugnahmen auch in Debatten, die den Bereichen „Energie“, „Wirtschaft“, „Außenpolitik“, „Recht" und „Raumordnung“ zugeteilt werden. Eine vergleichbare Entwicklung sieht man auch im Falle der Familienpolitik. Waren diese Bezugnahmen 2006 noch in der Rubrik „Gesellschaft/Bevölkerung“ konzentriert, tritt danach eine Verlagerung dieser Bezüge in den Bereich „Schulen“ auf.

Die Familienpolitik ist dabei zugleich ein interessanter Fall für eine Verschiebung des Schwerpunkts der Bezugnahmen im Vergleich der Jahre. In 2006 war „Familienpolitik“" schwerpunktmäßig Thema in Debatten des Bereichs „Gesellschaft/Bevölkerung", in 2007 verlagerte sich dieser Schwerpunkt in den schulischen Bereich. In 2009 wiederum treten die höchsten Häufigkeiten im Bereich des öffentlichen Haushalts auf. Diese Fluktuation führt bei der Kalkulation des HHI zu dem beachtenswerten Effekt, dass die HHI-Werte für jedes einzelne Jahr über dem Indexwert liegen, der sich ergibt, wenn der HHI für die gesamte Periode berechnet wird: Die Zeitreihenkurve verläuft über der im Diagramm gepunktet eingezeichneten horizontalen Linie, welche das Niveau des HHI-Werts für die gesamte Periode kennzeichnet. Wenn derartige Bezugnahmen zu unterschiedlichen Perioden jeweils in einem Bereich konzentriert erfolgen, das jedoch in unterschiedlichen Bereichen, so kann der Wert des Index für den gesamten Zeitraum niedriger sein als für die einzelnen Perioden. Ein solcher Fluktuationseffekt tritt neben der Familienpolitik in der Haushalts-, Finanz- und Sozialpolitik auf - also genau in jenen Feldern, die bei der Reihung der Felder nach ihren HHI-Werten die niedrigsten Werte aufweisen. Blasendiagramme (Abb. 5) zeigen im Zusammenspiel mit der Verlaufskurve des HHI den Zusammenhang, der zuvor abstrakt erläutert worden ist: In den genannten Fällen erfolgen die Häufungen der Bezugnahmen zu unterschiedlichen Zeitpunkten in verschiedenen Bereichen, so dass sich in mehreren einzelnen Perioden eine höhere Konzentration, aber insgesamt eine hohe Streuung ergeben kann. 
Abbildung 5: Finanz-, Haushalts- und Sozialpolitik als fluktuierende Felder

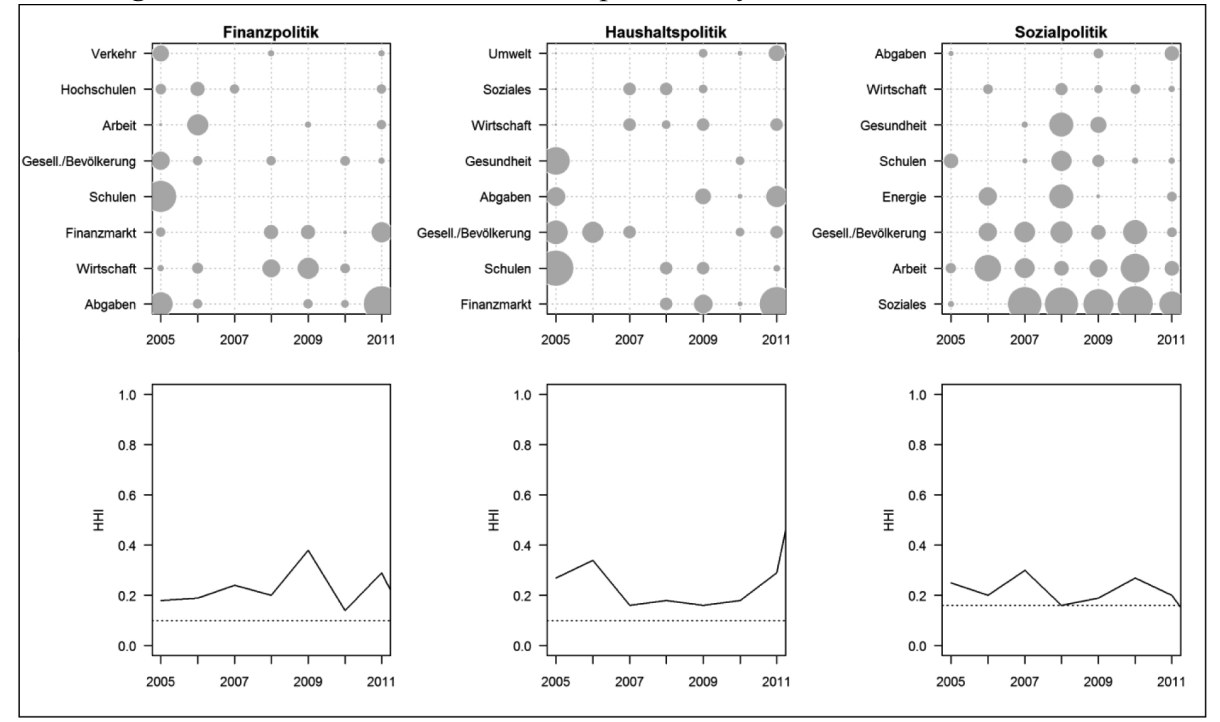

Quelle: Eigene Darstellung.

Im Fall der Sozialpolitik sticht ein besonders niedriger Konzentrationswert in 2008 ins Auge. Dem Blasendiagramm kann entnommen werden, dass zwar beim Lexem „Sozialpolitik“ die absolut häufigsten Werte in den Debatten der Rubrik ,Soziales“ erzielt werden $(\mathrm{tf}=12)$. Aber abgesehen davon werden mindestens fünf Bezüge zu „Sozialpolitik“ in immerhin vier weiteren Debattenkategorien hergestellt. In absteigender Reihenfolge sind dies (Häufigkeit jeweils in Klammern) die Kategorien „Schulen“(8), „Energie“(8), „Gesellschaft/Bevölkerung“(6) sowie Gesundheit (5).

\section{c) Textorientierte Detailanalyse: Sozialpolitische Grenzprozesse des Jahres 2008}

Anhand der fluktuierenden Feldbezüge der Sozialpolitik in den nordrhein-westfälischen Landtagsdebatten des Jahres 2008 soll nun gezeigt werden, wie die korpusunterstützte Analyse über eine Erfassung von Strukturen in großen Materialmengen den Blick hin zu einer Untersuchung mit größerer Tiefenschärfe anhand einzelner, konkreter Textpassagen führt und damit ein „Verstehen“ der quantifizierend er- 
reichten Befunde fördert. ${ }^{32}$ Zunächst ist es natürlich keineswegs überraschend, dass vor allem in Debatten der Rubrik ,Soziales“ über „Sozialpolitik“ gesprochen wurde. Auch im Jahr 2008 wurde dabei noch intensiv über die Reform der sozialen Sicherungssysteme (,Hartz IV“) debattiert. Bezugspunkt der Debatte sind konkrete Issues. Es wurden insbesondere die Folgen pauschalisierter Heizkosten für die Bezieher von Leistungen nach SGB II sowie die Frage der Rechtssicherheit für SGB II-Leistungsbezieher diskutiert. ${ }^{33}$ Die Verknüpfung mit der Sozialpolitik erfolgt auch bei den Debatten der Rubrik „Gesellschaft/Bevölkerung“ über Issues; hier über die Themen der Pflege (als Schwerpunkt der Sozialpolitik, PlPr 14/87) und der Rechte von Menschen mit Behinderung (PIPr 14/108). Beim Bereich der "Gesundheit" wird dahingehend die Suchtbekämpfung als Frage der Sozialpolitik diskutiert (PIPr 14/84). Zugleich erfolgt eine nicht issuebezogene, sondern allgemeine Verknüpfung von Fragen der Gesundheit mit Sozialpolitik über die Formel der „Gesundheits- und Sozialpolitik“. Dabei handelt es sich um eine gängige Engführung der Politikbereiche der Gesundheits- und Sozialpolitik ( $\mathrm{tf}=12 \mathrm{im}$ gesamten Korpus), die in Nordrhein-Westfalen auch regelmäßig zu einer Verbindung dieser Bereiche in einem Ministerium geführt hatte. Dies erklärt das Auftreten des Markers „Sozialpolitik“ in den Debatten der Rubrik „Gesundheit“.

Bemerkenswert ist nun die Verknüpfung von Sozial- und Schulpolitik. Diese erfolgt ausschließlich über Aussagen zum allgemeinen Verhältnis dieser beiden Felder zueinander, nicht anhand konkreter Issues. Dies erfolgt über formelhafte Formulierungen wie „Bildungspolitik ist Wirtschafts- und Sozialpolitik“ (Bernhard Recker [CDU], PIPr 14/104) beziehungsweise „Bildung für alle ist die nachhaltigste Wirtschafts- und Sozialpolitik" (Bernhard Tenhumberg [CDU], PIPr 14/107). Die Konstruktionen der Art „Bildung/Bildungspolitik ... ist ... Sozialpolitik“ finden sich dabei im zeitlichen Verlauf während der 14. Wahlperiode zunächst nur bei Sprechern der CDU und der FDP. Die Aussage stellt einen Zusammenhang zwischen Bildungs- und Sozialpolitik her, doch nicht im Sinne eines Erfordernis einer Vernetzung und kontinuierlichen Koordination. Tatsächlich entlastet eine gezielt gestaltete Bildungspolitik eine Sozialpolitik, deren Ziel die Bekämpfung sozialer Randlagen ist. Dieser Gehalt der Verknüpfung von Bildungs- und Sozialpolitik wird

32 Technisch ist dies bei der Korpusanalyse ohne weiteres realisierbar, weil die als System zur Korpusverwaltung genutzt Corpus Workbench nicht nur eine effiziente quantifizierende Analyse unterstützt, sondern jederzeit einen flexiblen Zugriff auf den Volltext - einschließlich aller Metainformationen zu einer Textpassage - ermöglicht.

33 So bei den Tagesordnungspunkten „Kommunen müssen sich an Recht und Gesetz halten - Heizkosten dürfen nicht über Pauschalen bei den SGB II-Beziehenden zu weiteren Einschnitten beim Existenzminimum führen“ (PIPr 14/96), „Drei Jahre SGB II in NRW“(PIPr 14/109) sowie „Schutz des Rechtsstaates muss auch für SGB II-Leistungsbezieher erhalten bleiben!“( $\operatorname{PIPr} 14 / 108)$. 
schließlich auch bei Übernahme jener „Bildungspolitik ist Sozialpolitik“-Formel deutlich, die ab 2011 bei Sprechern der SPD auftritt. „Gute Bildung ... ist die beste Sozialpolitik“ - so der SPD-Fraktionsvorsitzende Norbert Römer im Rahmen einer Haushaltsdebatte im Dezember 2011 (PIPr 15/50). Im Sinne der Konzeption der „präventiven“ bzw. „vorsorgenden“ Sozialpolitik hat sich die SPD eine Bestimmung der Interdependenz von Bildungs- und Sozialpolitik angeeignet mit dem Ziel, Bildungspolitik so zu akzentuieren, dass die Erforderlichkeit sozialer Ausgaben sinkt.

Im Bereich der Debatten der Kategorie „Energie“ erfolgt die Bezugnahme auf „Sozialpolitik“ über die sozialen Folgen hoher Energiepreise. Im Unterschied zur diskutierten Verknüpfung von Bildungs- und Sozialpolitik bleibt es allerdings strittig, wie das Verhältnis richtig zu bestimmen sei. Ein Konsens zeichnet sich im Untersuchungszeitraum nicht ab. Während Sprecher der SPD die Formel „Energiepolitik ... ist ... Sozialpolitik“"34 nutzen, kritisieren Sprecher von CDU und FDP, dass ein Eindringen sozialpolitischer Erwägungen in die Energiepolitik deren marktwirtschaftliche Logik gefährde. Wie es Christian Weisbrich (CDU) formuliert: „In der Energiepolitik müssen wir uns ordnungspolitisch, marktwirtschaftlich sauber verhalten. Entstehende Schwierigkeiten können und müssen wir über die Sozialpolitik ausgleichen. Wir dürfen aber nicht die Fachpolitik verhunzen." (PIPr 14/97) Nach dieser umstrittenen Logik soll Energiepolitik über marktwirtschaftliche Mechanismen Versorgungssicherheit und bezahlbaren Strom gewährleisten, während die Sozialpolitik einen möglicherweise erforderlichen sozialen Ausgleich zu schaffen hat. Im Fall des Verhältnisses von Sozial- und Bildungspolitik wurde die Sozialpolitik zum Leitstern der Bildungspolitik, in der Energiepolitik findet sich ein Widerstreit unterschiedlicher konzeptioneller Vorstellungen, wie das Verhältnis der Felder richtig zu bestimmen sei. Die richtige Bestimmung der Grenzen zwischen Energie- und Sozialpolitik bleibt in Folge von Überlappungen strittig.

Die Wechselwirkungen zwischen Feldern, auf welche der HHI als rein frequenzbasiertes Konzentrationsmaß und die Blasendiagramme als Visualisierung von Konzentration und Streuung hinweisen, können also, wie die qualitativ-deutende Auseinandersetzung mit den Belegstellen für die Suchterme zeigt, unterschiedlicher Art sein. Zwei unterschiedliche Umstände des „extraterritorialen“ Auftretens eines Feldmarkers außerhalb eines Kerngebiets konnten festgestellt werden: Erstens gab es die Fälle, in denen konkreten Issues eine Bedeutung im Kontext eines zusätzli-

34 Uwe Leuchtenberg (SPD): „Energiepolitik ist aber auch Standortpolitik, Familienpolitik, Wohnungsbaupolitik und vor allem Sozialpolitik. “(PlPr 14/26) Ähnliche Formulierungen des Sprechers finden sich in PIPr 14/28 und PIPr 14/82. Analog hierzu André Stinka in PIPr 14/134. 
chen Policy-Feldes zugewiesen wurde. Beispiele für den Feldbezug per Issue sind die Definition von Suchtbekämpfung oder der rechtlichen Gleichstellung von Behinderten als Sozialpolitik (PIPr 14/84). Zweitens treten die Feldmarker in allgemeinen programmatischen Bestimmungen des Verhältnisses von Policy-Feldern auf, wobei hier Fälle einer konzeptionellen Überwölbung unterschieden werden können von Bekräftigungen einer für erforderlich erachteten Differenzierungsstruktur. Die Bedeutung der Zahlen, welche Grundlage der Indikatorenberechnung und der Visualisierung sind, erschließt sich mit der qualitativen Analyse.

\section{Die korpusunterstützte Bewältigung der Flut digitaler Texte}

Policy-Felder sind nicht hermetisch gegeneinander abgegrenzt. Das Ausgangsproblem, dass man immer wieder grenzüberschreitende Prozesse beobachtet, dass einigen Politikbereichen „Querschnittscharakter“ zugeschrieben wird, wurde hier in einen systematischeren Befund überführt. Grenzüberschreitungen sind kein punktuelles Phänomen. Die hier verfolgte Erkundung auf Basis eines Plenardebattenkorpus mit allen Debatten im nordrhein-westfälischen Landtag der 14. und 15. Wahlperiode stellt zwar lediglich eine Untersuchung des parlamentarischen Sprachgebrauchs eines Bundeslandes dar. Gleichwohl konnten Erkenntnisse zu den diskursiven Konfigurationen von Policy-Feldern gewonnen werden. Auf der Grundlage der Frequenzen von Feldmarkern in Subkorpora, die entsprechend einer Systematik des nordrhein-westfälischen Landtags gebildet wurden, konnten für insgesamt 24 Policy-Felder Hirschman-Herfindahl-Indizes (HHI) berechnet werden. Die Felder wurden damit hinsichtlich des Grades ihrer Konzentration vergleichbar. Das Ergebnis dieser Vermessung: Policy-Felder unterscheiden sich hinsichtlich des Grades Ihrer Konzentration. Feldern mit einer stärkeren Konzentration stehen stärker streuende gegenüber, wobei Streuung keineswegs am stärksten in solchen Bereichen auftritt, für die ein „Querschnittscharakter" reklamiert wird. Der (deskriptive) Befund begründet gleichwohl, wie notwendig eine kritische Beschäftigung mit den mit dem Begriff des Politikfelds verbundenen Ausdifferenzierungsannahmen ist.

Für stark dezentrierte Felder konnte durch einen Vergleich der HHI-Niveaus für einzelne Jahre mit dem Fluktuationseffekt ein Phänomen bestimmt werden, das im Falle der Sozialpolitik - eine Bewegung von den Zahlen zurück zum Text vollziehend - näher untersucht wurde. Für überlappende Policy-Felder oder solche Felder, die durch ihren Querschnittscharakter dezentriert sind, lässt sich vorläufig feststellen: Die Instabilität der Bezugnahmen auf Felder mit unklar definierten Grenzen, ihr gelegentliches Eindringen in die Beratung von Materien anderer Felder, aber 
dann auch ihr Schwinden aus der Aufmerksamkeit - insgesamt Instabilität, Wanderung und Fluktuation solcher Felder -, darauf gibt die Auswertungen deutliche Hinweise. Phänomene der Fluktuation verdienen im Sinne einer Beschäftigung mit der „Politik der Aufmerksamkeit“ (Jones/Baumgartner 2005) und den Auswirkungen von Mehrdeutigkeit bzw. Ambiguität (Zahariadis 2003; Rüb 2008) im politischen Prozess noch nähere Beachtung.

Zielsetzung der korpusunterstützten Politikforschung war es hier nicht, deduktiv hergeleitete Hypothesen zu überprüfen. Die Quantifizierung dient vielmehr in explorativer Absicht der Aufdeckung von Mustern in Materialmengen, welche mit bloßem Auge nicht erkennbar sind. Dies hat eine Bedeutung, welche über die Beschäftigung mit der konkreten Forschungsfrage hinausreicht: Im Zeitalter der Digitalisierung entstehen ungeheure Fluten von Textdaten. Weil diese aber als Korpora aufbereitet maschinell verarbeitbar sind, eröffnen sich der Politikforschung Möglichkeiten, mittels Textstatistik Muster in dieser Materialflut zu erkennen. Die quantifizierende Auswertung lenkt die Aufmerksamkeit auf Zusammenhänge, die näher qualitativ zu untersuchen, zu interpretieren und zu entschlüsseln sind. Korpusunterstützte Politikforschung nutzt Textstatistik, hält sich aber den Weg zurück zum Text stets offen. Zugleich muss klar sein, wo die Grenzen und Entwicklungserfordernisse der korpusunterstützten Politikforschung als Forschungsansatz liegen: Das Korpus mit den Debatten im Landtag Nordrhein-Westfalen der 14. und 15. Wahlperiode ist zwar kein kleines Korpus, es umfasst immerhin einen Zeitraum von 7 Jahren und fast 15 Millionen Token. Die Uneindeutigkeit von Trends zeigt aber, dass der zeitliche Umfang des hier genutzten Korpus doch noch zu gering ist, um Aussagen über Veränderungen der Konzentration eines Policy-Feldes machen zu können. Policy-Wandel vollzieht sich oft in kurz gedrängten Umbruchphasen (Baumgartner/Jones 1993) - die Untersuchung größerer Zeiträume von einer Dekade und mehr (Sabatier 1993) bleibt allerdings erforderlich. Eine Verbreiterung der Datenbasis im Sinne einer Erfassung eines größeren Zeitraums erhöht die Relevanz des Plenardebattenkorpus als politikwissenschaftliche Datenbasis. ${ }^{35}$

35 Im Rahmen des Projekts „PolMine“ werden neben den nordrhein-westfälischen Plenardebatten die Plenardebatten aller anderen Landesparlamente sowie des Bundestags als Korpus aufbereitet. Das Ziel ist ein Korpus mit allen Debatten der Parlamente auf Bundes- und Landesebene seit 2000 (vgl. www.polmine.de). Damit entstehen Möglichkeiten des Bundesländervergleichs und des Vergleichs zwischen politischen Ebenen. Es entsteht eine neue Datengrundlage für die Parlamentarismusforschung allgemein, für Forschungsarbeiten zu politischen Dynamiken im Mehrebenensystem - etwa zu Prozessen der Diffusion und Konvergenz - oder zur Messung von Policy-Positionen. 
Korpora sind ein herausforderndes Material, sie stellen eine nicht geringe Herausforderung für das Forschungsdatenmanagement dar. ${ }^{36}$ Fragen des Forschungsdatenmanagements müssen technisch bewältigt werden, sie müssen aber im Dienste der Beschäftigung mit inhaltlich interessanten Fragen stehen. Aus der Untersuchung der Muster grenzüberschreitender Policy-Felder ergibt sich ein Bedarf, in der Computerlinguistik gängige Methoden der Textklassifikation einzusetzen, um eine von den Klassifikationen der Landtagsverwaltungen unabhängige Klassifikation zu gewinnen. Felder werden nicht nur, wie hier vereinfachend angenommen wurde, über Feldmarker wie „Integrationspolitik“ indiziert. Feldframes werden auch über komplexere sprachliche Muster aktiviert. Zur Beschaffenheit solcher Muster bietet die Korpuslinguistik theoretische Konzepte und Möglichkeiten an, wie diese sprachtechnologisch ermittelt werden können (Scharloth/Eugster/Bubenhofer i. E.).

Das diesem Beitrag zugrunde liegende Korpus lässt sich nach dem Kriterium der Parteizugehörigkeit von Sprechern partitionieren, so dass Fragen zur politischen Auseinandersetzung um Felddefinitionen bearbeitet werden können. Hier wurde zunächst mit dem Instrumentarium der korpusunterstützten Politikforschung der Nachweis erbracht, dass Grenzüberschreitungen weit mehr sind als eine Anomalie. Die Annahme der Ausdifferenzierung des Politikfelds - womöglich der Begriff des Politikfelds selbst - ist demnach neu zu bedenken. Korpora eröffnen bei einer überwältigenden Materialflut Möglichkeiten, modifizierten theoretischen Konzepten empirisch nachzugehen.

\section{Literatur}

Baumgartner, Frank R./Jones, Bryan D., 1993: Agendas and Instability in American Politics, Chicago.

Beckert, Jens, 1999: Agency, Entrepreneurs, and Institutional Change. The role of Strategic Choice and Institutionalized Practices in Organizations, in: Organization Studies 20 (5), 777-799.

Benford, Robert D./Snow, David A., 2000: Framing Processes and Social Movements. An Overview and Assessment, in: Annual Review of Sociology 26, 611-639.

Bird, Steven/Klein, Ewan/Loper, Edward, 2009: Natural Language Processing with Python, Sebastopol.

36 Datenumfang und Datenformat erfordern etwa spezielle Systeme zur Korpusverwaltung, die für einen Nutzerkreis nicht mehr auf dem lokalen Rechner des Forschers betrieben werden, sondern die als Forschungsinfrastruktur Server- bzw. Cloud-basiert zur Verfügung steht. 
Blätte, Andreas/Berenz, Silvia, 2012: PolMine. Korpusunterstützte Politikforschung. Dokumentation, http://polmine.sowi.uni-due.de/PolMine_Dokumentat ion.pdf (Stand: 5.9.2012).

Blum, Sonja/Schubert, Klaus, 2008: Politikfeldanalyse. Wiesbaden.

Bogdanor, Vernon (Hrsg.), 2005: Joined-Up Government, Oxford.

Bönker, Frank, 2008: Interdependenzen zwischen Politikfeldern. Die vernachlässigte sektorale Dimension der Politikverflechtung, in: Frank Janning/Katrin Toens (Hrsg.), Die Zukunft der Policy-Forschung. Theorien, Methoden, Anwendungen, Wiesbaden, 315-330.

Bourdieu, Pierre, 1998: Praktische Vernunft. Zur Theorie des Handelns, Frankfurt a. M.

Brier, Alan/Hopp, Bruno, 2010: Computer assisted Text Analysis in the Social Sciences, in: Quality and Quantity 45 (1), 103-128.

Bubenhofer, Noah, 2009: Sprachgebrauchsmuster. Korpuslinguistik als Methode der Diskurs- und Kulturanalyse, Berlin.

Burstein, Paul, 1991: Policy Domains. Organization, Culture, and Policy Outcomes, in: Annual Review of Sociology 17, 327-350.

Christensen, Tom/Laegreid, Per, 2007: The Whole-of-Government Approach to Public Sector Reform, in: Public Administration Review 67 (6), 1059-1066.

DiMaggio, Paul/Powell, Walter W., 1983: The Iron Cage Revisited. Institutional Isomorphism and Collective Rationality in Organizational Fields, in: American Sociological Review 48 (2), 147-160.

Fischer, Frank, 2003: Reframing public policy. Discursive politics and deliberative practices, Oxford.

Fischer, Frank/Forester, John (Hrsg.), 1993: The Argumentative Turn in Policy Analysis and Planning, Durham/London.

Fligstein, Neil, 2001: Institutional Entrepreneurs And Cultural Frames. The Case of the European Union's Single Market Program, in: European Societies 3 (3), 261-288.

Fligstein, Neil/McAdam, Doug, 2012: A Theory of Fields, Oxford.

Früh, Werner, 2011: Inhaltsanalyse. Theorie und Praxis, Stuttgart (7. Aufl.).

Glasze, Georg, 2007: Vorschläge zur Operationalisierung der Diskurstheorie von Laclau und Mouffe in einer Triangulation von lexikometrischen und interpretativen Methoden, in: Forum: Qualitative Sozialforschung 8 (2), http://www.qual itative-research.net/index.php/fqs/article/download/239/530 (Stand: 5.9.2012).

Janning, Frank, 2011: Die Spätgeburt eines Politikfeldes. Die Institutionalisierung der Verbraucherschutzpolitik in Deutschland und im internationalen Vergleich, Baden-Baden. 
Jantz, Bastian/Veit, Sylvia, 2011: Steuerung von Querschnittspolitik durch das Bundeskanzleramt. Das Beispiel Bürokratieabbau, in: Martin Florack/Timo Grunden (Hrsg.), Regierungszentralen. Organisation, Steuerung und Politikformulierung zwischen Formalität und Informalität, Wiesbaden, 285-310.

Jochim, Ashley E./May, Peter J., 2010: Beyond Subsystems. Policy Regimes and Governance, in: The Policy Studies Journal 38 (2), 303-327.

Jones, Bryan D./Baumgartner, Frank R., 2005: The Politics of Attention. How Government prioritizes Problems, Chicago.

Jones, Michael D./Jenkins-Smith, Hank C., 2009: Trans-Subsystem Dynamics. Policy Topography, Mass Opinion, and Policy Change, in: Policy Studies Journal 37 (1), 37-58.

Krippendorff, Klaus, 2009: Content analysis. An Introduction to its Methodology, Thousand Oaks (2. Aufl.).

Lakoff, George/Wehling, Elisabeth, 2008: Auf leisen Sohlen ins Gehirn. Politische Sprache und ihre heimliche Macht, Heidelberg.

Langtangen, Hans Petter, 2011: A Primer on Scientific Programming with Python, Berlin (2. Aufl.).

Laver, Michael/Benoit, Kenneth/Garry, John, 2003: Extracting Policy Positions from Political Texts Using Words as Data, in: American Political Science Review 97 (2), 311-331.

Ling, Tom, 2002: Delivering Joined-Up Government in the UK. Dimensions, Issues and Problems, in: Public Administration 80 (4), 615-642.

May, Peter J./Jochim, Ashley E./Sapotichne, Joshua, 2011: Constructing Homeland Security. An Anemic Policy Regime, in: Policy Studies Journal 39 (2), 285-307. May, Peter J./Jones, Bryan D./Beem, Betsi B./Neff-Sharum, Emily A./Poague, Melissa K., 2005: Policy Coherence and Component-Driven Policymaking. Arctic Policy in Canada and the United States, in: Policy Studies Journal 33 (1), 37-63. May, Peter J./Sapotichne, Joshua/Workman, Samuel, 2006: Policy Coherence and Policy Domains, in: Policy Studies Journal 34 (3), 381-403.

McCool, Daniel, 1998: The Subsystem Family of Concepts. A Critique and a Proposal, in: Political Research Quarterly 51 (2), 551-570.

Nullmeier, Frank/Rüb, Friedbert W., 1993: Die Transformation der Sozialpolitik. Vom Sozialstaat zum Sicherungsstaat, Frankfurt a. M./New York.

Pappi, Franz Urban/Schmitt, Ralf/Linhart, Eric, 2008: Die Ministeriumsverteilung in den deutschen Landesregierungen seit dem Zweiten Weltkrieg, in: Zeitschrift für Parlamentsfragen (2), 323-342.

Pollitt, Christopher, 2003: Joined-up Government. A Survey, in: Political Studies Review 1, 34-49. 
Reckwitz, Andreas, 2008: Unscharfe Grenzen. Perspektiven der Kultursoziologie, Bielefeld.

Robertson, David, 1976: A Theory of Party Competition, London.

Roe, Emery, 1994: Narrative Policy Analysis. Theory and Practice, Durham u. a.

Rüb, Friedbert W., 2008: Policy-Analyse unter den Bedingungen von Kontingenz.

Konzeptionelle Überlegungen zu einer möglichen Neuorientierung, in: Frank Janning/Katrin Toens (Hrsg.), Die Zukunft der Policy-Forschung. Theorien, Methoden, Anwendungen, Wiesbaden, 88-111.

Sabatier, Paul A., 1993: Policy Change over a Decade or More, in: Paul A. Sabatier/ Hank C. Jenkins-Smith (Hrsg.), Policy Change and Learning. An Advocacy Coalition Approach, Boulder, 13-39.

Scharloth, Joachim/Eugster, David/Bubenhofer, Noah, i. E.: Das Wuchern der Rhizome. Linguistische Diskursanalyse und Data-driven Turn, in: Dietrich Busse/ Wolfgang Teubert (Hrsg.), Linguistische Diskursanalyse - Neue Perspektiven, Wiesbaden.

Scharpf, Fritz W., 2000: Interaktionsformen. Akteurzentrierter Institutionalismus in der Politikforschung, Opladen.

Scheufele, Bertram, 2003: Frames - Framing - Framing-Effekte. Theoretische und methodische Grundlegung des Framing-Ansatzes sowie empirische Befunde zur Nachrichtenproduktion, Wiesbaden.

Schimank, Uwe, 1985: Der mangelnde Akteursbezug systemtheoretischer Erklärungen gesellschaftlicher Differenzierung. Ein Diskussionsvorschlag, in: Zeitschrift für Soziologie 14 (6), 421-434.

Schimank, Uwe, 2007: Theorien gesellschaftlicher Differenzierung, Wiesbaden (3. Aufl.).

Schneider, Volker/Janning, Frank, 2006: Politikfeldanalyse. Akteure, Diskurse und Netzwerke in der öffentlichen Politik, Wiesbaden.

Schröter, Melani/Carius, Björn, 2008: Vom politischen Gebrauch der Sprache: Wort, Text, Diskurs. Eine Einführung, Frankfurt a. M.

Schubert, Klaus von/Bandelow, Nils C. (Hrsg.), 2009: Lehrbuch der Politikfeldanalyse 2.0, München (2. Aufl.).

Slapin, Jonathan B./Proksch, Sven-Oliver, 2008: A Scaling Model for Estimating Time-Series Party Positions from Texts, in: American Journal of Political Science 52 (3), 705-722.

Tremmel, Jörg, 2011: Von der,sozialen Gerechtigkeit` zur,Generationengerechtigkeit‘? Eine Analyse der Bundestagsdokumente 2005 und 2009, in: Zeitschrift für Parlamentsfragen 42 (4), 691-707. 
Zahariadis, Nikolaos, 2003: Ambiguity and Choice in Public Policy. Political Decision Making in modern Democracies, Washington D.C.

Züll, Cornelia/Mohler, Peter, 1992: Textanalyse. Anwendungen der computergestützten Inhaltsanalyse, Opladen.

Korrespondenzanschrift:

Prof. Dr. Andreas Blätte

Juniorprofessur für Politikwissenschaft der WestLB-Stiftung Zukunft NRW

NRW School of Governance

Institut für Politikwissenschaft

Universität Duisburg-Essen

Lotharstraße 53, LS 019

47057 Duisburg

E-Mail: andreas.blaette@uni-due.de

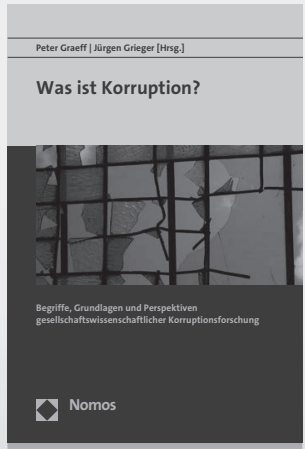

\section{Was ist Korruption?}

Begriffe, Grundlagen und Perspektiven gesell-

schaftswissenschaftlicher Korruptionsforschung

Herausgegeben von Peter Graeff und

Jürgen Grieger

2012, 244 S., brosch., 26,- $€$

ISBN 978-3-8329-7341-4

Weitere Informationen: www.nomos-shop.de/14530

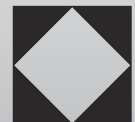

